\title{
Sumsets of reciprocals in prime fields and multilinear Kloosterman sums
}

\author{
J. Bourgain \\ Institute for Advanced Study, \\ Princeton, NJ 08540, USA \\ bourgain@ias.edu \\ M. Z. Garaev \\ Centro de Ciencias Matemáticas, \\ Universidad Nacional Autónoma de México, \\ Morelia 58089, Michoacán, México \\ garaev@matmor.unam.mx
}

\begin{abstract}
We obtain new results on additive properties of the set

$$
I^{-1}=\left\{x^{-1}: \quad x \in I\right\}
$$

where $I$ is an arbitrary interval in the field of residue classes modulo a large prime $p$. We combine our results with multilinear exponential sum estimates and obtain new results on incomplete multilinear Kloosterman sums.
\end{abstract}




\section{Introduction}

In what follows, $\varepsilon>0$ is an arbitrary fixed constant, $\mathbb{F}_{p}$ is the field of residue classes modulo a large prime $p$ which frequently will be associated with the set $\{0,1, \ldots, p-1\}$. Given an integer $x$ coprime to $p$ (or an element $x$ from $\left.\mathbb{F}_{p}^{*}=\mathbb{F}_{p} \backslash\{0\}\right)$ we use $x^{*}$ or $x^{-1}$ to denote its multiplicative inverse modulo $p$.

Let $I$ be a non-zero interval in $\mathbb{F}_{p}$. Additive properties of the reciprocalset

$$
I^{-1}=\left\{x^{-1}: \quad x \in I\right\},
$$

with a subsequent application to Kloosterman sums have been considered in [5]. Among other results, it has been shown there that for any $\delta>0$ there exists $k \in \mathbb{Z}_{+}$such that the sumset

$$
k\left(I^{-1}\right)=\left\{x_{1}^{-1}+\ldots+x_{k}^{-1}: \quad x_{i} \in I\right\}
$$

satisfies

$$
\left|k\left(I^{-1}\right)\right|>p^{-\delta} \min \left\{|I|^{2}, p\right\} .
$$

In the most interesting case $|I|<p^{1 / 2}$ this implies that $\left|k\left(I^{-1}\right)\right|>|I|^{2} p^{-\delta}$. From some recent results in [12] (see Lemma 10 below) it follows that

$$
\left|I^{-1}+I^{-1}\right|>\min \left\{|I|^{2}, \sqrt{p|I|}\right\}|I|^{o(1)} .
$$

In particular, if $|I|<p^{1 / 3}$, then

$$
\left|I^{-1}+I^{-1}\right|>|I|^{2+o(1)} .
$$

The aim of the present paper is to establish new additive properties of the set $I^{-1}$. We then combine our results with recent estimates of multilinear exponential sum bounds from [6] and obtain new results on multilinear Kloosterman sums.

The structure of the paper is as follows. In section 2 we state our results on additive properties of the set $I^{-1}$ and on estimates of Kloosterman sums. In section 3 we give some basic preliminaries which are used throughout the paper. In sections $4-8$ we give some backgrounds and prove preliminary lemmas. The proof of our results on additive properties of reciprocals on intervals (Theorems 1-6) is given in section 9. The proof of Theorems 7-13 are given in section 10. In section 11 we give the proof of Theorem 14 on Archimedian counterpart of Karatsuba's estimate, in section 12 we give the 
proof of Theorem 15 on $\pi(x)-\pi(x-y)$, Theorem 16 on a linear Kloosterman sums and Theorem 17 on Brun-Titchmarsh theorem.

In this paper we consider only the case of prime modulus. The case of composite modulus will be considered in a forthcoming paper.

\section{Statement of results}

\section{$2.1 \quad$ Reciprocals of intervals}

We recall that $I$ denotes an arbitrary non-zero interval in $\mathbb{F}_{p}$. We first start with results on additive properties of $I^{-1}$.

Theorem 1. For any fixed positive integer constant $k$ the number $J_{2 k}$ of solutions of the congruence

$$
x_{1}^{-1}+\ldots+x_{k}^{-1}=x_{k+1}^{-1}+\ldots+x_{2 k}^{-1}, \quad x_{1}, \ldots, x_{2 k} \in I,
$$

satisfies

$$
J_{2 k}<\left(|I|^{2 k^{2} /(k+1)}+\frac{|I|^{2 k}}{p}\right)|I|^{o(1)} .
$$

Recall that (2) is equivalent to saying that for any $\varepsilon>0$ there exists $c=c(k ; \varepsilon)>0$ such that

$$
J_{2 k}<c\left(|I|^{2 k^{2} /(k+1)}+\frac{|I|^{2 k}}{p}\right)|I|^{\varepsilon} .
$$

Corollary 1. Let $|I|<p^{1 / 2}$. Then for any fixed positive integer constant $k$,

$$
\left|k\left(I^{-1}\right)\right|>|I|^{2 k /(k+1)+o(1)} .
$$

We remark that for $k=3$ one can prove the bound

$$
\left|I^{-1}+I^{-1}+I^{-1}\right|>|I|^{1.55+o(1)} .
$$

We next consider a ternary additive congruence with $I^{-1}$.

Theorem 2. Let $|I|<p^{3 / 46}$. Then for any element $\lambda \in \mathbb{F}_{p}$ with

$$
\lambda \notin I^{-1} \cup\{0\},
$$


the number $J$ of solutions of the congruence

$$
x^{-1}+y^{-1}+z^{-1}=\lambda, \quad x, y, z \in I,
$$

satisfies

$$
J<|I|^{2 / 3+o(1)} .
$$

The restriction (3) is motivated by the possibility of $|I|^{1+o(1)}$ solutions otherwise (for instance, $z=\lambda^{-1}$ and $x+y=0$ ).

From Theorem 2 it easily follows that for $|I|<p^{3 / 46}$, one has the bound

$$
\left|I^{-1}+I^{-1}+I^{-1}\right|>|I|^{7 / 3+o(1)} .
$$

The following statements show that for sufficiently small $I$ one has optimal bounds.

Theorem 3. Let $|I|<p^{1 / 18}$. Then the number $J_{6}$ of solutions of the congruence

$$
x_{1}^{-1}+x_{2}^{-1}+x_{3}^{-1}=x_{4}^{-1}+x_{5}^{-1}+x_{6}^{-1}, \quad x_{1}, \ldots, x_{6} \in I,
$$

satisfies

$$
J_{6}<|I|^{3+o(1)} .
$$

In particular, for $|I|<p^{1 / 18}$ we have

$$
\left|I^{-1}+I^{-1}+I^{-1}\right|>|I|^{3+o(1)} .
$$

Theorem 4. There is an absolute constant $c>0$ such that for any fixed positive integer constant $k$ and any interval $I \subset \mathbb{F}_{p}$ with $|I|<p^{c / k^{2}}$ the number $J_{2 k}$ of solutions of the congruence

$$
x_{1}^{-1}+\ldots+x_{k}^{-1}=x_{k+1}^{-1}+\ldots+x_{2 k}^{-1}, \quad x_{1}, \ldots, x_{2 k} \in I,
$$

satisfies

$$
J_{2 k}<|I|^{k+o(1)} .
$$

In particular, for such intervals I we have

$$
\left|k\left(I^{-1}\right)\right|>|I|^{k+o(1)} .
$$

Remark 1. From the proof it is clear that in Theorem 4 one can take $c=1 / 4$. 
Theorem 5. Let $I=[1, N]$. Then the number $J_{2 k}$ of solutions of the congruence

$$
x_{1}^{*}+\ldots+x_{k}^{*} \equiv x_{k+1}^{*}+\ldots+x_{2 k}^{*} \quad(\bmod p), \quad x_{1}, \ldots, x_{2 k} \in I,
$$

satisfies

$$
J_{2 k}<(2 k)^{90 k^{3}}(\log N)^{4 k^{2}}\left(\frac{N^{2 k-1}}{p}+1\right) N^{k} .
$$

We also give a version of Theorem 5 , where the variables $x_{j}$ are restricted to prime numbers. By $\mathcal{P}$ we denote the set of primes.

Theorem 6. Let $I=[1, N]$. Then the number $J_{2 k}$ of solutions of the congruence

$$
x_{1}^{*}+\ldots+x_{k}^{*} \equiv x_{k+1}^{*}+\ldots+x_{2 k}^{*} \quad(\bmod p), \quad x_{1}, \ldots, x_{2 k} \in I \cap \mathcal{P},
$$

satisfies

$$
J_{2 k}<(2 k)^{k}\left(\frac{N^{2 k-1}}{p}+1\right) N^{k}
$$

\subsection{Incomplete multilinear Kloosterman sums}

Below we use the abbreviation $e_{p}(z)=e^{2 \pi i z / p}$. The incomplete Kloosterman sums

$$
\sum_{x=M+1}^{M+N} e_{p}\left(a x^{*}+b x\right)
$$

where $a$ and $b$ are integers, $\operatorname{gcd}(a, p)=1$, are well known in the literature, with a variety of applications. These sums are estimated by $O\left(p^{1 / 2} \log p\right)$ as a consequence of Weil bounds. For $M=0$ and $N$ very small (that is, $\left.N=p^{o(1)}\right)$ these sums have been estimated by Korolev [26].

The incomplete bilinear Kloosterman sums

$$
S=\sum_{x_{1}=M_{1}+1}^{M_{1}+N_{1}} \sum_{x_{2}=M_{2}+1}^{M_{2}+N_{2}} \alpha_{1}\left(x_{1}\right) \alpha_{2}\left(x_{2}\right) e_{p}\left(a x_{1}^{*} x_{2}^{*}\right),
$$

where $\alpha_{i}\left(x_{i}\right) \in \mathbb{C},\left|\alpha_{i}\left(x_{i}\right)\right| \leq 1$, are also well known in the literature. Observe, that if one of the parameters $N_{i}$ is much larger than $p^{1 / 2}$, then $S$ can easily 
be estimated. For instance, if, say, $N_{1}^{1-c}>p^{1 / 2}$ for some $c>0$, one can use the Weil bound and get

$$
\begin{aligned}
|S|^{2} \leq & N_{1} \sum_{x_{1}=M_{1}+1}^{M_{1}+N_{1}}\left|\sum_{x_{2}=M_{2}+1}^{M_{2}+N_{2}} \alpha_{2}\left(x_{2}\right) e_{p}\left(a x_{1}^{*} x_{2}^{*}\right)\right|^{2} \leq \\
& N_{1} \sum_{y=M_{2}+1}^{M_{2}+N_{2}} \sum_{z=M_{2}+1}^{M_{2}+N_{2}} \mid \sum_{x_{1}=M_{1}+1}^{M_{1}+N_{1}} e_{p}\left(a x_{1}^{*}\left(y^{*}-z^{*}\right) \mid \ll N_{1}^{2} N_{2}+N_{1} N_{2}^{2} \sqrt{p}(\log p),\right.
\end{aligned}
$$

which implies that

$$
|S|<\left(N_{2}^{-1 / 2}+N_{1}^{-c / 2}\right)\left(N_{1} N_{2}\right)^{1+o(1)} .
$$

Thus, the most nontrivial case is $N_{i}<p^{1 / 2}$. When $M_{1}=M_{2}=0$ the sum $S$ (in a more general form in fact) has been estimated by Karatsuba [24, 25] for very short ranges of $N_{1}$ and $N_{2}$, and by Bourgain [5] for arbitrary $M_{1}, M_{2}$ provided that $N_{1} N_{2}>p^{1 / 2+\varepsilon}$. A full explicit version of Bourgain's result has been given by Baker [2].

The incomplete $n$-linear Kloosterman sums

$$
\sum_{x_{1}=M_{1}+1}^{M_{1}+N_{1}} \ldots \sum_{x_{n}=M_{n}+1}^{M_{n}+N_{n}} e_{p}\left(a_{1} x_{1}+\ldots+a_{n} x_{n}+a_{n+1}\left(x_{1} \ldots x_{n}\right)^{*}\right),
$$

where $a_{i} \in \mathbb{Z}, \operatorname{gcd}\left(a_{n+1}, p\right)=1$, have been studied by Luo [28] and Shparlinski [30] for arbitrary $n$. The main tool they used are the bounds of Burgess [11] on incomplete Gauss sums.

Here, we combine our Theorems 1, 3, 4,5 with the multilinear exponential sum bounds from [6] (see Lemma 1 below) and obtain new estimates on Kloosterman sums. In what follows, $\alpha_{1}\left(x_{1}\right), \ldots, \alpha_{n}\left(x_{n}\right)$ are arbitrary complex numbers with $\left|\alpha_{i}\left(x_{i}\right)\right| \leq 1$.

Theorem 7. For any intervals $I_{1}, I_{2}$ with

$$
\left|I_{1}\right|>p^{1 / 18}, \quad\left|I_{2}\right|>p^{5 / 12+\varepsilon}
$$

we have

$$
\max _{(a, p)=1}\left|\sum_{x_{1} \in I_{1}} \sum_{x_{2} \in I_{2}} \alpha_{1}\left(x_{1}\right) \alpha_{2}\left(x_{2}\right) e_{p}\left(a x_{1}^{*} x_{2}^{*}\right)\right|<p^{-\delta}\left|I_{1}\right|\left|I_{2}\right|
$$

for some $\delta=\delta(\varepsilon)>0$. 
Note that $\left|I_{1}\right|\left|I_{2}\right|=p^{1 / 2-1 / 36+\varepsilon}$.

Remark 2. The statement of Theorem 7 remains true for the general sum

$$
\sum_{x_{1}=1}^{N_{1}} \sum_{x_{2}=1}^{N_{2}} \alpha_{1}\left(x_{1}\right) \alpha_{2}\left(x_{2}\right) e_{p}\left(a x_{1}^{*} x_{2}^{*}+b x_{1} x_{2}\right) .
$$

This can be achieved incorporating [5, Lemma A.8].

When $M_{1}=M_{2}=0$, we prove the following result, expanding the range of applicability of Karatsuba's estimate [24].

Theorem 8. Let $I_{1}=\left[1, N_{1}\right], I_{2}=\left[1, N_{2}\right]$. Then uniformly over all positive integers $k_{1}, k_{2}$ and $\operatorname{gcd}(a, p)=1$ we have

$$
\begin{aligned}
\left|\sum_{x_{1} \in I_{1}} \sum_{x_{2} \in I_{2}} \alpha_{1}\left(x_{1}\right) \alpha_{2}\left(x_{2}\right) e_{p}\left(a x_{1}^{*} x_{2}^{*}\right)\right|<\left(2 k_{1}\right)^{\frac{45 k_{1}^{2}}{k_{2}}}\left(2 k_{2}\right)^{\frac{45 k_{2}^{2}}{k_{1}}}(\log p)^{2\left(\frac{k_{1}}{k_{2}}+\frac{k_{2}}{k_{1}}\right)} \times \\
\times\left(\frac{N_{1}^{k_{1}-1}}{p^{1 / 2}}+\frac{p^{1 / 2}}{N^{k_{1}}}\right)^{1 /\left(2 k_{1} k_{2}\right)}\left(\frac{N_{2}^{k_{2}-1}}{p^{1 / 2}}+\frac{p^{1 / 2}}{N^{k_{2}}}\right)^{1 /\left(2 k_{1} k_{2}\right)} N_{1} N_{2} .
\end{aligned}
$$

Given $N_{1}, N_{2}$ we choose $k_{1}, k_{2}$ such that

$$
N_{1}^{2\left(k_{1}-1\right)}<p \leq N_{1}^{2 k_{1}}, \quad N_{2}^{2\left(k_{2}-1\right)}<p \leq N_{2}^{2 k_{2}}
$$

and the bound will be nontrivial unless both $N_{1}, N_{2}$ are within $p^{\varepsilon}$-ratio of an element of $\left\{p^{\frac{1}{2 l}}, l \in \mathbb{Z}_{+}\right\}$. Thus, we have the following

Corollary 2. Let $I_{1}=\left[1, N_{1}\right], I_{2}=\left[1, N_{2}\right]$, where for $i=1$ or $i=2$

$$
N_{i} \notin \bigcup_{j \geq 1}\left[p^{\frac{1}{2 j}-\varepsilon}, p^{\frac{1}{2 j}+\varepsilon}\right] .
$$

Then

$$
\max _{(a, p)=1}\left|\sum_{x_{1}=1}^{N_{1}} \sum_{x_{2}=1}^{N_{2}} \alpha_{1}\left(x_{1}\right) \alpha_{2}\left(x_{2}\right) e_{p}\left(a x_{1}^{*} x_{2}^{*}\right)\right|<p^{-\delta} N_{1} N_{2}
$$

for some $\delta=\delta(\varepsilon)>0$. 
Theorem 9. Let $I_{1}, I_{2} \subset \mathbb{F}_{p}$ be intervals of sizes $N_{1}, N_{2}$ in arbitrary position. Then

$$
\begin{aligned}
& \max _{(a, p)=1}\left|\sum_{x_{1} \in I_{1}} \sum_{x_{2} \in I_{2}} \alpha_{1}\left(x_{1}\right) \alpha_{2}\left(x_{2}\right) e_{p}\left(a x_{1}^{*} x_{2}^{*}\right)\right| \ll \\
& \ll p^{1 / 8} N_{1}^{3 / 4} N_{2}^{3 / 4}\left(\frac{N_{1}^{3}}{p}+1\right)^{1 / 16}\left(\frac{N_{2}^{3}}{p}+1\right)^{1 / 16} .
\end{aligned}
$$

Some relevant to Theorem 9 results with intervals starting from the origin can be found in [2], [15], [19].

Theorem 10. Let $k_{1}, k_{2}$ be positive integer constants, $I_{1}, I_{2} \subset \mathbb{F}_{p}$ be intervals of sizes $N_{1}, N_{2}$ in arbitrary position and

$$
N_{1}<p^{\frac{k_{1}+1}{2 k_{1}}}, \quad N_{2}<p^{\frac{k_{2}+1}{2 k_{2}}} .
$$

Then

$$
\begin{aligned}
& \max _{(a, p)=1}\left|\sum_{x_{1} \in I_{1}} \sum_{x_{2} \in I_{2}} \alpha_{1}\left(x_{1}\right) \alpha_{2}\left(x_{2}\right) e_{p}\left(a x_{1}^{*} x_{2}^{*}\right)\right| \\
&\left(p^{\frac{1}{2 k_{1} k_{2}}} N_{1}^{-\frac{1}{k_{2}\left(k_{1}+1\right)}} N_{2}^{-\frac{1}{k_{1}\left(k_{2}+1\right)}}\right)\left(N_{1} N_{2}\right)^{1+o(1)} .
\end{aligned}
$$

We next consider multilinear Kloosterman sums.

Theorem 11. Let $n \geq 7$ and $N^{n}>p^{1 / 3+\varepsilon}$. Then for any intervals $I_{1}, \ldots, I_{n}$ of length $N$ we have

$$
\max _{(a, p)=1}\left|\sum_{x_{1} \in I_{1}} \ldots \sum_{x_{n} \in I_{n}} \alpha_{1}\left(x_{1}\right) \ldots \alpha_{n}\left(x_{n}\right) e_{p}\left(a x_{1}^{*} \ldots x_{n}^{*}\right)\right|<p^{-\delta} N^{n}
$$

for some $\delta=\delta(\varepsilon, n)>0$.

Theorem 12. There exists an absolute constant $C>0$ such that for any positive integer $n$ and any intervals $I_{1}, \ldots, I_{n}$ of length $N$ with $N>p^{C / n^{2}}$, we have

$$
\max _{(a, p)=1}\left|\sum_{x_{1} \in I_{1}} \ldots \sum_{x_{n} \in I_{n}} \alpha_{1}\left(x_{1}\right) \ldots \alpha_{n}\left(x_{n}\right) e_{p}\left(a x_{1}^{*} \ldots x_{n}^{*}\right)\right|<p^{-\delta} N^{n}
$$

for some $\delta=\delta(n)>0$. 
Remark 3. It can be proved that Theorem 12 holds with $C=4$. This can be done using the geometry of numbers in the style of [9] to get a suitable for this version of our Theorem 4 .

Theorem 13. Let $I_{1}, \ldots, I_{n}$ be intervals in $[1, p-1]$ with

$$
\left|I_{1}\right| \cdots\left|I_{n}\right|>p^{1 / 2+\varepsilon} .
$$

Then we have

$$
\max _{(a, p)=1}\left|\sum_{x_{1} \in I_{1}} \ldots \sum_{x_{n} \in I_{n}} \alpha_{1}\left(x_{1}\right) \ldots \alpha_{n}\left(x_{n}\right) e_{p}\left(a x_{1}^{*} \ldots x_{n}^{*}\right)\right|<p^{-\delta}\left|I_{1}\right| \ldots\left|I_{n}\right|
$$

for some $\delta=\delta(\varepsilon, n)>0$.

There is the following 'Archimedian' counterpart of the Karatsuba estimate.

Theorem 14. Let $\xi \in \mathbb{R}$ with $|\xi|>N_{1} N_{2}$ and $k_{1}, k_{2} \in \mathbb{Z}_{+}$. Then

$$
\left|\sum_{\substack{n n_{1} \sim N_{1} \\ n_{2} \sim N_{2}}} e^{i \frac{1}{n_{1}} \frac{1}{n_{2}} \xi}\right|<c\left(k_{1}, k_{2}, \varepsilon\right) \gamma\left(N_{1} N_{2}\right)^{1+\varepsilon}
$$

with

$\gamma=\left\{\left(\frac{|\xi|}{N_{1} N_{2}} N_{1}^{-2 k_{1}}+\frac{N_{1} N_{2}}{|\xi|} N_{1}^{2\left(k_{1}-1\right)}\right)\left(\frac{|\xi|}{N_{1} N_{2}} N_{2}^{-2 k_{2}}+\frac{N_{1} N_{2}}{|\xi|} N_{2}^{2\left(k_{2}-1\right)}\right)\right\}^{1 /\left(4 k_{1} k_{2}\right)}$

Given $|\xi|>N_{1} N_{2}$, choose $k_{1}, k_{2}$ satisfying

$$
N_{i}^{2\left(k_{i}-1\right)} \leq \frac{|\xi|}{N_{1} N_{2}}<N_{i}^{2 k_{i}} .
$$

Then each factor in expression for $\gamma$ in Theorem 14 is $O(1)$.

Exponential sums of the type (4) appear, for instance, in the proof of Theorem 13.8 in [18]

$$
\pi(x)-\pi(x-y) \leq(2-\delta) \frac{y}{\log y}, \quad x^{\theta}<y<x,
$$

where, as usual, $\pi(z)$ is the number of primes not exceeding $z$ and $\delta=\delta(\theta)>$ 0 . Here $\theta>0$ may be small, $x$ is sufficiently large in terms of $\theta$. In [18] the proof of (5) is based on estimates of exponential sums of the form $\sum_{n \sim N} e\left(\frac{\xi}{n}\right)$ using either Weil or Vinogradov, when $\theta$ is very small. Using Theorem 14 one gets a better estimate. 
Theorem 15. The estimate (5) holds with

$$
\delta<\frac{2(1-\theta)}{12\left(\theta^{-1}+1\right)\left(\theta^{-1}+0.5\right)+1-\theta} \sim \theta^{2} .
$$

We shall apply trilinear exponential sum bounds from [6] (see Lemma 1 below) to a linear Kloosterman sums and Brun-Titchmarsh theorem.

Theorem 16. The following bound holds:

$$
\max _{(a, p)=1}\left|\sum_{n \leq N} e_{p}\left(a n^{*}\right)\right| \ll \frac{(\log \log p)^{3} \log p}{(\log N)^{3 / 2}} N,
$$

where the implied constant is absolute.

It follows that if $N=p^{\varepsilon}$ with $\varepsilon$ fixed, the saving is $O\left((\log \log p)^{3} /(\log p)^{1 / 2}\right)$ and the estimate is nontrivial if $N>\exp \left((\log p)^{\frac{2}{3}}(\log \log p)^{3}\right)$. This improves some results of Korolev [26] in the case of prime moduli. We also refer the reader to [27] for some variants of the problem.

We remark that in [23] it is claimed that if $\varepsilon>0$ is fixed, then for $p^{\varepsilon}<N<p^{4 / 7}$ one has the bound

$$
\left|\sum_{n=1}^{N} e_{p}\left(a n^{*}\right)\right|<\frac{N}{(\log N)^{1-\varepsilon}},
$$

but the proof given there is in doubt.

For $(a, q)=1, \pi(x ; q, a)$ denotes the number of primes $p \leq x, p \equiv a$ $(\bmod q)$. We aim to improve the result of Friedlander-Iwaniec on $\pi(x ; q, a)$ as follows:

Theorem 17. Let $q=x^{\theta}$, where $\theta<1$ is close to 1 . Then

$$
\pi(x ; q, a)<\frac{c x}{\phi(q) \log \frac{x}{q}}
$$

with $c=2-c_{1}(1-\theta)^{2}$, for some absolute constant $c_{1}>0$ and all sufficiently large $x$ in terms of $\theta$.

The constant $c_{1}$ is effective and can be made explicit. 


\section{Preliminaries}

Throughout the paper we will use well-known connections between the number of solutions of symmetric equations and the cardinality of corresponding set. Let $T$ be the number of solutions of the equation

$$
x_{1}+\ldots+x_{n}=y_{1}+\ldots+y_{n}
$$

where for each $i$ the variables $x_{i}, y_{i}$ run through a set $A_{i}$. Then for any subset

$$
\Omega \subset A_{1} \times \ldots \times A_{n}
$$

one has the bound

$$
\left|\left\{x_{1}+\ldots+x_{n}:\left(x_{1}, \ldots x_{n}\right) \in \Omega\right\}\right| \geq \frac{|\Omega|^{2}}{T} .
$$

This estimate follows from the observation that if $T_{n}(\Omega ; \lambda)$ is the number of solutions of the equation

$$
x_{1}+\ldots+x_{n}=\lambda, \quad\left(x_{1}, \ldots, x_{n}\right) \in \Omega,
$$

and

$$
X=\left\{x_{1}+\ldots+x_{n}:\left(x_{1}, \ldots x_{n}\right) \in \Omega\right\},
$$

then

$$
T \geq \sum_{\lambda \in X} T_{n}(\Omega ; \lambda)^{2} \geq \frac{1}{|X|}\left|\sum_{\lambda \in X} T_{n}(\Omega ; \lambda)\right|^{2}=\frac{|\Omega|^{2}}{|X|} .
$$

In particular,

$$
\left|A_{1}+\ldots+A_{n}\right|=\#\left\{a_{1}+\ldots+a_{n}: a_{i} \in A_{i}\right\} \geq \frac{\left|A_{1}\right|^{2} \ldots\left|A_{n}\right|^{2}}{T} .
$$

We note that if $A_{1}, \ldots, A_{2 n} \subset \mathbb{F}_{p}$ and $T_{2 n}(\lambda)$ is the number of solutions of the congruence

$$
x_{1}+\ldots+x_{2 n} \equiv \lambda \quad(\bmod p), \quad\left(x_{1}, \ldots, x_{2 n}\right) \in A_{1} \times \ldots \times A_{2 n},
$$

then

$$
T_{2 n}(\lambda) \leq\left(J_{1} \ldots J_{2 n}\right)^{\frac{1}{2 n}}
$$


where $J_{i}$ is the number of solutions of the congruence

$$
y_{1}+\ldots+y_{n} \equiv y_{k+1}+\ldots+y_{2 k} \quad(\bmod p), \quad y_{1}, \ldots, y_{2 k} \in A_{i} \text {. }
$$

Indeed, we have

$$
T=\frac{1}{p} \sum_{a=0}^{p-1} \sum_{x_{1} \in A_{1}} \ldots \sum_{x_{2 n} \in A_{2 n}} e_{p}\left(a x_{1}\right) \ldots e_{p}\left(a x_{2 n}\right) e_{p}(-a \lambda) .
$$

Applying Hölder's inequality we get

$$
T \leq \prod_{j=1}^{n}\left(\frac{1}{p} \sum_{a=0}^{p-1}\left|\sum_{x_{j} \in A_{j}} e_{p}\left(a x_{j}\right)\right|^{2 n}\right)^{\frac{1}{2 n}}=\left(J_{1} \ldots J_{2 n}\right)^{\frac{1}{2 n}} .
$$

In proofs of some of our results we will use the observation that if $X, Y \in$ $\mathbb{F}_{p}$, then the number of solutions of the congruence equation

$$
\frac{1}{y+x_{1}}+\ldots+\frac{1}{y+x_{n}}=\frac{1}{y+x_{n+1}}+\ldots+\frac{1}{y+x_{2 n}}, \quad x_{i} \in X, y \in Y,
$$

is at most $O\left(|X|^{n} Y+|X|^{2 n}\right)$, the implied constant may depend only on $n$. Indeed, the contribution from those $\left(x_{1}, \ldots, x_{2 n}\right) \in X^{2 n}$ for which the series $x_{1}, \ldots, x_{2 n}$ contains at most $n$ distinct elements, is $O\left(|X|^{n}|Y|\right)$. On the other hand, if there are more than $n$ distinct elements in this series, then we can assume that $x_{1} \notin\left\{x_{2}, \ldots, x_{2 n}\right\}$. For each such given $\left(x_{1}, \ldots, x_{2 n}\right) \in X^{2 n}$ the polynomial

$$
P(Z)=\prod_{i \neq 1}\left(Z+x_{i}\right)+\ldots+\prod_{i \neq n}\left(Z+x_{i}\right)-\prod_{i \neq n+1}\left(Z+x_{i}\right)-\ldots-\prod_{i \neq 2 n}\left(Z+x_{i}\right)
$$

is nonzero (as $P\left(-x_{1}\right) \neq 0$ ) and since $P(y)=0$ we get at most $2 n-1$ possibilities for $y$. See also [2, Lemmas 2,3] for more general statements.

\section{Multilinear exponential sums}

The following result, which we state as a lemma, has been proved by Bourgain [6]. It is based on results from additive combinatorics, in particular sum-product estimates. This lemma will be used in the proof of our results on Kloosterman sums. 
Lemma 1. Let $\gamma_{1}\left(x_{1}\right), \ldots, \gamma_{n}\left(x_{n}\right)$ be non-negative real numbers satisfying

$$
\left\|\gamma_{i}\right\|_{1}=\sum_{x=0}^{p-1}\left|\gamma_{i}(x)\right| \leq 1, \quad\left\|\gamma_{i}\right\|_{2}=\left(\sum_{x=0}^{p-1}\left|\gamma_{i}(x)\right|^{2}\right)^{1 / 2}<p^{-\delta} .
$$

Assume further

$$
\prod_{i=1}^{n}\left\|\gamma_{i}\right\|_{2}<p^{-1 / 2-\delta}
$$

where $0<\delta<1 / 4$. Then there is the exponential sum bound

$$
\left|\sum_{x_{1}=0}^{p-1} \ldots \sum_{x_{n}=0}^{p-1} \gamma_{1}\left(x_{1}\right) \ldots \gamma_{n}\left(x_{n}\right) e_{p}\left(x_{1} \ldots x_{n}\right)\right|<p^{-\delta^{\prime}}
$$

with some $\delta^{\prime}>(\delta / n)^{C n}$.

\section{Resultant Bound}

We shall need the following resultant bound from [9].

Lemma 2. Let $N \geq 1, \sigma, \vartheta \in \mathbb{R}$, and let $m, n \geq 2$ be fixed integers. Assume also that one of the following conditions hold:

(i) $\sigma \geq 0$;

(ii) $\vartheta \geq 0$;

(iii) $\sigma+\vartheta \geq-1$.

Let $P_{1}(Z)$ and $P_{2}(Z)$ be non-constant polynomials with integer coefficients,

$$
P_{1}(Z)=\sum_{i=0}^{m-1} a_{i} Z^{m-1-i}, \quad P_{2}(Z)=\sum_{i=0}^{n-1} b_{i} Z^{n-1-i}
$$

such that

$$
\begin{aligned}
& \left|a_{i}\right|<A N^{i+\sigma}, \quad i=0, \ldots, m-1 \\
& \left|b_{i}\right|<A N^{i+\vartheta}, \quad i=0, \ldots, n-1
\end{aligned}
$$

for some $A$. Then

$$
\operatorname{Res}\left(P_{1}, P_{2}\right) \ll N^{(m-1+\sigma)(n-1+\vartheta)-\sigma \vartheta},
$$

where the implicit constant in $\ll$ depends only on $A, m$ and $n$. 


\section{Background on geometry of numbers}

We need some facts from the geometry of numbers. Recall that a lattice in $\mathbb{R}^{n}$ is an additive subgroup of $\mathbb{R}^{n}$ generated by $n$ linearly independent vectors. Take an arbitrary convex compact and symmetric with respect to 0 body $D \subset \mathbb{R}^{n}$. Recall that, for a lattice $\Gamma \subset \mathbb{R}^{n}$ and $i=1, \ldots, n$, the $i$-th successive minimum $\lambda_{i}(D, \Gamma)$ of the set $D$ with respect to the lattice $\Gamma$ is defined as the minimal number $\lambda$ such that the set $\lambda D$ contains $i$ linearly independent vectors of the lattice $\Gamma$. Obviously, $\lambda_{1}(D, \Gamma) \leq \ldots \leq \lambda_{n}(D, \Gamma)$. We need the following result given in [3, Proposition 2.1] (see also [31, Exercise 3.5.6] for a simplified form that is still enough for our purposes).

Lemma 3. We have

$$
|D \cap \Gamma| \leq \prod_{i=1}^{n}\left(\frac{2 i}{\lambda_{i}(D, \Gamma)}+1\right) .
$$

Denoting, as usual, by $(2 n+1)$ !! the product of all odd positive numbers up to $2 n+1$, we get the following

Corollary 3. We have

$$
\prod_{i=1}^{n} \min \left\{\lambda_{i}(D, \Gamma), 1\right\} \leq \frac{(2 n+1) ! !}{|D \cap \Gamma|} .
$$

\section{$7 \quad$ Equations with many variables}

The following lemma is due to Karatsuba [24].

Lemma 4. The following bound holds:

$$
\begin{gathered}
\left|\left\{\left(x_{1}, \ldots, x_{2 k}\right) \in[1, N]^{2 k}: \frac{1}{x_{1}}+\ldots+\frac{1}{x_{k}}=\frac{1}{x_{k+1}}+\ldots+\frac{1}{x_{2 k}}\right\}\right| \\
<(2 k)^{80 k^{3}}(\log N)^{4 k^{2}} N^{k} .
\end{gathered}
$$

The following elementary statement will be used to exclude some degenerated cases in the proof of Lemma 6 below. 
Lemma 5. Let $c \in \mathbb{C}, c_{1}, \ldots, c_{r} \in \mathbb{C}^{*}, S$ be a finite subset of $\mathbb{C}$. Let $T_{r}$ be the number of solutions of the equation

$$
c_{1} x_{1}+\ldots+c_{r} x_{r}=c, \quad x_{1}, \ldots, x_{r} \in S
$$

and $J_{2 s}$ be the number of solutions of the equation

$$
x_{1}+\ldots+x_{s}=x_{s+1}+\ldots+x_{2 s}, \quad x_{1}, \ldots, x_{2 s} \in S .
$$

If $r=2 k$ for some integer $k$, then $T_{2 k} \leq J_{2 k}$. If $r=2 k-1$ for some integer $k$, then $T_{2 k-1}^{2} \leq J_{2 k-2} J_{2 k}$.

Proof. Let $r=2 k$. Among all $2 k+1$-tuples $\left(l_{1}, \ldots, l_{2 k}, l\right)$ with

$$
l_{i} \in\left\{ \pm c_{1}, \ldots, \pm c_{2 k}\right\}, \quad l \in\{0, c\}
$$

we consider the one for which the number of solutions of the equation

$$
l_{1} x_{1}+\ldots+l_{2 k} x_{2 k}=l, \quad x_{1}, \ldots, x_{2 k} \in S,
$$

is maximal. There can be several $2 k+1$-tuples with this property. We choose the one for which the sequence

$$
l_{1}, \ldots, l_{2 k}
$$

contains the maximal number of elements from $\left\{-l_{1}, l_{1}\right\}$. We fix one such $\left(l_{1}, \ldots, l_{2 k}, l\right)$ with

$$
l_{i} \in\left\{-l_{1}, l_{1}\right\}, \quad i=1, \ldots, s,
$$

such that either $s=2 k$ or $l_{t} \notin\left\{-l_{1}, l_{1}\right\}$ for $t>s$. Denote by $L_{2 k}$ the number of solutions of (6), that is

$$
l_{1} x_{1}+\ldots+l_{k} x_{k}=l-\left(l_{k+1} x_{k+1}+\ldots+l_{2 k} x_{2 k}\right) ; \quad x_{1}, \ldots, x_{2 k} \in S .
$$

Note that

$$
L_{2 k}=\sum_{\lambda} I_{1}(\lambda) I_{2}(\lambda)
$$

where $I_{1}(\lambda)$ is the number of solutions of the equation

$$
l_{1} x_{1}+\ldots+l_{k} x_{k}=\lambda, \quad x_{1}, \ldots, x_{k} \in S
$$


and $I_{2}(\lambda)$ is the number of solutions of the equation

$$
l-\left(l_{k+1} x_{k+1}+\ldots+l_{2 k} x_{2 k}\right)=\lambda ; \quad x_{k+1}, \ldots, x_{2 k} \in S .
$$

Applying the Cauchy-Schwarz inequality, we obtain

$$
L_{2 k}^{2} \leq\left(\sum_{\lambda} I_{1}^{2}(\lambda)\right)\left(\sum_{\lambda} I_{2}^{2}(\lambda)\right)
$$

The quantity in the second parenthesis is equal to the number of solutions of the equation

$$
l_{k+1} x_{1}+\ldots+l_{k} x_{k}=l_{k+1} x_{k+1}+\ldots+l_{2 k} x_{2 k}, \quad x_{1}, \ldots, x_{2 k} \in S .
$$

Hence, by the maximality of $L_{2 k}$ we have

$$
L_{2 k} \leq \sum_{\lambda} I_{1}^{2}(\lambda)
$$

The right hand side indicates the number of solutions of the equation

$$
l_{1} x_{1}+\ldots+l_{k} x_{k}=l_{1} x_{k+1}+\ldots+l_{k} x_{2 k}, \quad x_{1}, \ldots, x_{2 k} \in S .
$$

Clearly, the series

$$
l_{1}, \ldots, l_{k}, l_{1}, \ldots, l_{k}
$$

contains $\min \{2 s, 2 k\}$ elements from $\left\{-l_{1}, l_{1}\right\}$. Hence, by the maximality of $s$ we have $s=2 k$. Therefore, $l_{i} \in\left\{-l_{1}, l_{1}\right\}$ for all $i$, which implies that the number of solutions of the equation (7) is equal to $J_{2 k}$. Thus, $L_{2 k} \leq J_{2 k}$ implying $T_{2 k} \leq I_{2 k}$. This proves the first statement of the lemma.

To prove the second statement of our lemma, we write the corresponding to $T_{2 k-1}$ equation in the form

$$
c_{1} x_{1}+\ldots+c_{k} x_{k}=l-\left(c_{k+1} x_{k+1}+\ldots+c_{2 k-1} x_{2 k-1}\right)
$$

and, as before, apply the Cauchy-Schwarz inequality to get that

$$
T_{2 k-1}^{2} \leq\left(\sum_{\lambda} I_{11}^{2}(\lambda)\right)\left(\sum_{\lambda} I_{22}^{2}(\lambda)\right),
$$

where $I_{11}(\lambda)$ is the number of solutions of the equation

$$
c_{1} x_{1}+\ldots+c_{k} x_{k}=\lambda, \quad x_{1}, \ldots, x_{k} \in S
$$


and $I_{22}(\lambda)$ is the number of solutions of the equation

$$
-\left(c_{k+1} x_{k+1}+\ldots+c_{2 k-1} x_{2 k-1}\right)=\lambda, \quad x_{k+1}, \ldots, x_{2 k-1} \in S .
$$

Applying the first statement of our lemma we obtain

$$
\sum_{\lambda} I_{11}^{2}(\lambda) \leq J_{2 k} ; \quad \sum_{\lambda} I_{22}^{2}(\lambda) \leq J_{2 k-2}
$$

which finishes the proof of the lemma.

Let $\xi$ be an algebraic integer of degree $d$ and $O_{\mathbb{K}}$ be the ring of integers in $\mathbb{K}=\mathbb{Q}(\xi)$. In the proof of Lemma 6 below we use the language of ideals in the Dedekind domain $O_{\mathbb{K}}$. We refer the reader to [22, Chapter 12] and [4, Chapter 3] for a background material. Below, all considered ideals are integral. In particular, we say that an ideal $I_{2}$ divides $I_{1}$ if for some ideal $I_{3}$ we have $I_{1}=I_{2} I_{3}$.

We will use well-known properties of ideals. For instance, if $I_{1}$ and $I_{2}$ are ideals such that $I_{1} \subset I_{2}$ then $I_{2}$ divides $I_{1}$ (see, for example, [22, Proposition $12.2 .7])$.

Clearly, the uniqueness of factorization into prime ideals implies that if $I_{1}, I_{2}, I_{3}$ are ideals in $O_{\mathbb{K}}$ such that $I_{3}$ divides $I_{1} I_{2}$ then $I_{3}=J_{1} J_{2}$ for some ideals $J_{1}$ dividing $I_{1}$ and $J_{2}$ dividing $I_{2}$, respectively.

It is also useful to recall that the number of integral ideals in $O_{\mathbb{K}}$ of norm $n$ is at most $\tau(n)^{d}$, where $\tau$ is the divisor function. In particular, for fixed constant $d$ and large $n$ this is a quantity of size $n^{o(1)}$.

We recall that the logarithmic height of a nonzero polynomial $P \in \mathbb{Z}[Z]$ is defined as the maximum logarithm of the largest (by absolute value) coefficient of $P$. The logarithmic height of an algebraic number $\alpha$ is defined as the logarithmic height of its minimal polynomial. It is a well-known consequence of basic properties of Mahler's measure that if $P, Q \in \mathbb{Z}[Z]$ are two univariate non-zero polynomials with $Q \mid P$ and if $P$ is of logarithmic height at most $H$ then $Q$ is of logarithmic height at most $H+O(1)$, where the implied constant depends only on $\operatorname{deg} P$ (see, for example, [29, Theorem 4.2.2]).

In particular, it follows that if $P \in \mathbb{Z}[Z]$ is a nonconstant polynomial with coefficients bounded by $M$ (by absolute value), then every root $\sigma$ of $P(Z)$ can be represented in the form $\xi / q$ where $\xi$ is an algebraic integer of logarithmic height at most $O(\log M)$ and $q>0$ is an integer with $q<M^{O(1)}$, where the implied constants depend only on $\operatorname{deg} P$. 
Lemma 6. For any fixed positive integer constant $r$ and all values of $\sigma \in \mathbb{C}$ the number $T_{r}(\sigma, N)$ of solutions of the equation

$$
\frac{1}{\sigma+x_{1}}+\ldots+\frac{1}{\sigma+x_{r}}=\frac{1}{\sigma+x_{r+1}}+\ldots+\frac{1}{\sigma+x_{2 r}}
$$

in positive integers $x_{1}, \ldots, x_{2 r} \leq N$ satisfies

$$
T_{r}(a, N)<N^{r+o(1)} .
$$

Proof. For the brevity denote $T_{r}=T_{r}(a, N)$. We shall prove the lemma by induction on $r$. For $r=1$ the result is trivial. Let $r \geq 2$.

From Lemma 5 it follows that the number of solutions satisfying $x_{i}=x_{j}$ for some $i \neq j$ contributes to $T_{r}$ a quantity bounded by

$$
O\left(\sqrt{T_{r-1} T_{r}}\right)
$$

Thus, by the induction hypothesis it suffices to prove that

$$
T_{r}^{\prime}<N^{r+o(1)}
$$

where $T_{r}^{\prime}$ denotes the number of solutions with $x_{i} \neq x_{j}$ for all $i \neq j$. We can assume that $T_{r}^{\prime}>N^{r}$ as otherwise there is nothing to prove. Rewrite our equation in the form

$$
\prod_{i \neq 1}\left(\sigma+x_{i}\right)+\ldots+\prod_{i \neq r}\left(\sigma+x_{i}\right)=\prod_{i \neq r+1}\left(\sigma+x_{i}\right)+\ldots+\prod_{i \neq 2 r}\left(\sigma+x_{i}\right)
$$

and consider the polynomial $P(Z)$ defined as

$$
\prod_{i \neq 1}\left(Z+x_{i}\right)+\ldots+\prod_{i \neq r}\left(Z+x_{i}\right)-\prod_{i \neq r+1}\left(Z+x_{i}\right)-\ldots-\prod_{i \neq 2 r}\left(Z+x_{i}\right) .
$$

Clearly, $\operatorname{deg} P \leq 2 r-1$. Note also that $P\left(-x_{1}\right) \neq 0$, so $P(Z)$ is not a zero polynomial. Moreover, $P(\sigma)=0$, implying that $P(Z)$ is not a constant polynomial either. Therefore, we may assume that $\sigma$ is an algebraic number of degree $d$ with $1 \leq d \leq 2 r-1$ and logarithmic height $O(\log N)$. We can write $\sigma=\xi / q$, where $\xi$ is an algebraic integer of height $O(\log N)$ and $q$ is an integer with $q=N^{O(1)}$. Then our equation takes the form

$$
\prod_{i \neq 1}\left(\xi+q x_{i}\right)+\ldots+\prod_{i \neq r}\left(\xi+q x_{i}\right)=\prod_{i \neq r+1}\left(\xi+q x_{i}\right)+\ldots+\prod_{i \neq 2 r}\left(\xi+q x_{i}\right) .
$$


Let $O_{\mathbb{K}}$ be the ring of integers in $\mathbb{K}=\mathbb{Q}(\xi)$. The idea is to use the observation that for each $i=1, \ldots, 2 r$

$$
\xi+q x_{i} \quad \text { divides } q^{2 r-1} \prod_{j \neq i}\left(x_{j}-x_{i}\right) .
$$

The strategy to evaluate the number of solutions of (8) is to introduce consequently the variables $x_{1}, x_{2}, \ldots$ taking into account congruence conditions that appeared fixing previous variables.

Given an ideal $I$ we denote by $\nu(I)$ its norm. We factor the principal ideal $\left(\xi+q x_{1}\right)$ into factors

$$
\left(\xi+q x_{1}\right)=I_{1} J_{1}
$$

where the prime factors of $I_{1}$ divide $q$ and $\left(J_{1}, q\right)=1$. By $(9)$, the norm $\nu\left(J_{1}\right)$ divides $\prod_{j \geq 2}\left(x_{j}-x_{1}\right)^{d}$. Hence,

$$
x_{j} \equiv x_{1} \quad\left(\bmod r_{j}\right), \quad 2 \leq j \leq 2 r,
$$

for some $r_{j} \in \mathbb{Z}_{+}$such that $\nu\left(J_{1}\right) \mid \nu_{1}^{d}$ with $\nu_{1}=\prod_{j \geq 2} r_{j}$ and $\nu_{1} \mid \nu\left(J_{1}\right)$. We restrict $\nu_{1}$ to dyadic intervals, that is there exists a fixed number $\mu_{1} \geq 1$ such that if we restrict $\nu_{1}$ to the size range

$$
\mu_{1} \leq \nu_{1} \leq 2 \mu_{1},
$$

then the number of solutions of our equation with this restriction will be changed by at most $N^{o(1)}$ times.

For every $x_{1}$ we consider at most $N^{o(1)}$ different cases and in accordance to (10) specify $x_{j}, j \geq 2$, to arithmetic progressions $L_{2, j} \in[1, N]$, where thus

$$
\prod_{j \geq 2}\left|L_{2, j}\right|<\frac{N^{2 r-1+o(1)}}{\mu_{1}} .
$$

At the next step for $x_{2} \in L_{2,2}$ we factor

$$
\left(\xi+q x_{2}\right)=I_{2} J_{2},
$$

where the prime factors of $I_{2}$ divide $q$ or $\nu\left(\xi+q x_{1}\right)$ and $J_{2}$ is coprime to $q$ and $\nu\left(\xi+q x_{1}\right)$. Hence, $J_{2}$ is coprime to $\left(x_{2}-x_{1}\right)$ and again by (9), the norm 
$\nu\left(J_{2}\right)$ divides $\prod_{j \geq 3}\left(x_{j}-x_{2}\right)^{d}$. Arguing as before, we find $\nu_{2}$ such that $\nu\left(J_{2}\right) \mid \nu_{2}^{d}$ and $\nu_{2} \mid \nu\left(J_{2}\right)$. We can restrict $\nu_{2}$ to a size range, that is there exists a fixed number $\mu_{2} \geq 1$ (independent on variables $x_{i}$ ) such that if we restrict $\nu_{2}$ to

$$
\mu_{2} \leq \nu_{2} \leq 2 \mu_{2}
$$

then the number of solutions of our equation with this restriction will be changed by at most $N^{o(1)}$ times. For every $x_{2} \in L_{2,2}$ we can consider at most $N^{o(1)}$ possibilities and specify $x_{j}, j \geq 3$, to arithmetic progressions $L_{3, j} \subset L_{2, j}$ where

$$
\prod_{j \geq 3}\left|L_{3, j}\right| \leq \frac{1}{\mu_{2}} \prod_{j \geq 3}\left|L_{2, j}\right|, \quad \nu\left(J_{2}\right) \mid \nu_{2}^{d} .
$$

Note indeed that the progression $L_{2, j}$ are defined to some modulus $r_{j} \mid \nu_{1}$ and $\operatorname{gcd}\left(r_{j}, \nu\left(J_{2}\right)\right)=1$ since $\nu\left(J_{1}\right), \nu\left(J_{2}\right)$ are coprime.

At the next step for $x_{3} \in L_{3,3}$ we factor

$$
\left(\xi+q x_{3}\right)=I_{3} J_{3}
$$

where the prime factors of $I_{3}$ divides either $(q), \nu\left(\xi+q x_{1}\right)$ or $\nu\left(\xi+q x_{2}\right)$, and $J_{3}$ is coprime with $(q), \nu\left(\xi+q x_{1}\right)$ and $\nu\left(\xi+q x_{2}\right)$. We find $\nu_{3}$ similar to the previous cases and specify it to a size range $\mu_{3} \leq \nu_{3} \leq 2 \mu_{3}$, where $\mu_{3}$ is independent on variables. The continuation of the process is clear.

We now consequently fix $x_{1} \leq N, x_{2} \in L_{2,2}, \ldots, x_{2 r} \in L_{2 r, 2 r}$, that is, we fix $x_{1}$ and considering $N^{o(1)}$ possibilities for arithmetic progressions $L_{2, j}, j \geq 3$, we fix $x_{2} \in L_{2,2}$, then considering $N^{o(1)}$ possibilities for arithmetic progressions $L_{3, j}, j \geq 3$, we fix $x_{3} \in L_{3,3}$ and iterate this until we fix $x_{2 r} \in L_{2 r, 2 r}$. We estimate the number of solutions of (8) as $N^{o(1)}$ contributions of the form

$$
N\left|L_{2,2}\right|\left|L_{3,3}\right| \ldots\left|L_{2 r, 2 r}\right| \text {. }
$$

From (12), (14) and iteration we get

$$
\begin{gathered}
N^{2 r-1} \gtrsim \mu_{1}\left|L_{2,2}\right| \prod_{j \geq 3}\left|L_{2, j}\right| \\
\gtrsim \mu_{1} \mu_{2}\left|L_{2,2}\right|\left|L_{3,3}\right| \prod_{j \geq 4}\left|L_{3, j}\right| \\
\ldots \\
\gtrsim \mu_{1} \ldots \mu_{2 r-1}\left|L_{2,2}\right| \ldots\left|L_{2 r, 2 r}\right|
\end{gathered}
$$


Here $A \gtrsim B$ means $A>B N^{o(1)}$. Thus, the number of solutions of (8) may be bounded by

$$
\frac{N^{2 r+o(1)}}{\mu_{1} \ldots \mu_{2 r-1}} .
$$

Next, returning to our construction, it is clear that $\nu\left(I_{1}\right)=N^{O(1)}$ and since the prime factors of $I_{1}$ divide $q$, it follows that the number of possibilities for $I_{1}$ is at most $N^{o(1)}$. Fixing $I_{1}$ and denoting $\xi_{1}=\xi, \xi_{2}, \ldots, \xi_{d}$ the conjugates of $\xi$, we have

$$
\prod_{s=1}^{d}\left(\xi_{s}+q x_{1}\right)=\nu\left(I_{1}\right) \nu\left(J_{1}\right)
$$

and since $\nu\left(J_{1}\right) \mid \nu_{1}^{d}$ it follows that $\nu\left(J_{1}\right)$ is determined by $\nu_{1}$ with up to $N^{o(1)}$ possibilities. Thus, given $\nu_{1}$ we retrieve $x_{1}$ with up to $N^{o(1)}$ possibilities. It follows that in the size range (11) the number of possibilities for $x_{1}$ is at most $N^{o(1)} \mu_{1}$. Next, once $x_{1}$ is given, there are at most $N^{o(1)}$ possibilities for the ideal $I_{2}$ and similarly $N^{o(1)} \mu_{2}$ possibilities for $x_{2}$. It follows that the number of possibilities for $x_{1}, x_{2}, \ldots, x_{2 r-1}$ is at most $\mu_{1} \mu_{2} \ldots \mu_{2 r-1} N^{o(1)}$. Thus, the number of solutions of (8) is bounded by $\mu_{1} \mu_{2} \ldots \mu_{2 r-1} N^{o(1)}$. Since it is also bounded by (15), the result follows.

Lemma 7. Let $x, y, z, a_{1}, a_{2}, b_{1}, b_{2}$ be complex numbers such that

$$
\left\{\begin{array}{l}
x y z=a_{1}(x+y+z)+b_{1} \\
x y+y z+z x=a_{2}(x+y+z)+b_{2}
\end{array}\right.
$$

Then

$\left(x^{2}-a_{2} x+a_{1}\right)\left(y^{2}-a_{2} y+a_{1}\right)\left(z^{2}-a_{2} z+a_{1}\right)=\left(b_{1}-\alpha_{1} b_{2}-\alpha_{1}^{3}\right)\left(b_{1}-\alpha_{2} b_{2}-\alpha_{2}^{3}\right)$,

where

$$
\alpha_{1}=\frac{a_{2}+\sqrt{a_{2}^{2}-4 a_{1}}}{2}, \quad \alpha_{2}=\frac{a_{2}-\sqrt{a_{2}^{2}-4 a_{1}}}{2} .
$$

Proof. Indeed, since $\alpha_{i}^{2}-a_{2} \alpha_{i}+a_{1}=0$, we have

$$
\left(x-\alpha_{i}\right)\left(y-\alpha_{i}\right)\left(z-\alpha_{i}\right)=b_{1}-\alpha_{i} b_{2}-\alpha_{i}^{3}, \quad i=1,2 .
$$

Multiplying these equalities the claim follows. 
Lemma 8. Let $A, B$ be integers with $A B \neq 0$ and $|A|,|B|<N^{O(1)}$. Then the diophantine equation

$$
A x y+B x+B y=0
$$

has at most $N^{o(1)}$ solutions in integers $x, y$ with $|x|,|y| \leq N^{O(1)}$.

Proof. Indeed, we have

$$
(A x+B)(A y+B)=B^{2}
$$

and the statement follows from the well-known bound for the divisor function.

Lemma 9. Let $a_{0}, b_{0}, u_{0}, v_{0}$ be integers with $b_{0} u_{0} v_{0} \neq 0$ and

$$
\left|a_{0}\right|,\left|b_{0}\right|,\left|u_{0}\right|,\left|v_{0}\right|<N^{O(1)}
$$

Assume that

$$
\frac{u_{0}}{v_{0}} \notin\left\{\frac{b_{0}}{a_{0}+b_{0} x}: \quad 1 \leq x \leq N\right\} .
$$

Then the number $J$ of solutions of the diophantine equation

$$
\begin{aligned}
& u_{0}\left(a_{0}+b_{0} x_{1}\right)\left(a_{0}+b_{0} x_{2}\right)\left(a_{0}+b_{0} x_{3}\right)=v_{0} b_{0} \times \\
& \left(\left(a_{0}+b_{0} x_{1}\right)\left(a_{0}+b_{0} x_{2}\right)+\left(a_{0}+b_{0} x_{2}\right)\left(a_{0}+b_{0} x_{3}\right)+\left(a_{0}+b_{0} x_{3}\right)\left(a_{0}+b_{0} x_{1}\right)\right)
\end{aligned}
$$

in integers $x_{1}, x_{2}, x_{3}$ with

$$
1 \leq x_{i} \leq N, \quad a_{0}+b_{0} x_{i} \neq 0
$$

satisfies

$$
J<N^{2 / 3+o(1)} .
$$

Proof. We can clearly assume that

$$
b_{0}>0, \quad v_{0}>0, \quad \operatorname{gcd}\left(a_{0}, b_{0}\right)=1 .
$$

We observe that if one of the variables $x_{1}, x_{2}, x_{3}$ is determined, then for the rest two variables there remain at most $N^{o(1)}$ possibilities. Indeed, let $x_{1}$ be fixed. Then denoting

$$
X_{i}=a_{0}+b_{0} x_{i}, \quad A=u_{0} X_{1}-v_{0} b_{0}, \quad B=-v_{0} b_{0} X_{1},
$$


we get

$$
A X_{2} X_{3}+B X_{2}+B X_{3}=0 .
$$

By the condition, $A B \neq 0$ and $|A|,|B|<N^{O(1)}$. Hence by Lemma 8, we can retrieve $X_{2}, X_{3}$, and thus the numbers $x_{2}, x_{3}$, with at most $N^{o(1)}$ possibilities.

Denote

$$
u_{0}^{\prime}=\frac{u_{0}}{\operatorname{gcd}\left(u_{0}, b_{0} v_{0}\right)}, \quad \frac{b_{0} v_{0}}{\operatorname{gcd}\left(u_{0}, b_{0} v_{0}\right)}=w_{0} \geq 1
$$

We have

$$
\begin{aligned}
& u_{0}^{\prime}\left(a_{0}+b_{0} x_{1}\right)\left(a_{0}+b_{0} x_{2}\right)\left(a_{0}+b_{0} x_{3}\right)=w_{0} \times \\
& \left(\left(a_{0}+b_{0} x_{1}\right)\left(a_{0}+b_{0} x_{2}\right)+\left(a_{0}+b_{0} x_{2}\right)\left(a_{0}+b_{0} x_{3}\right)+\left(a_{0}+b_{0} x_{3}\right)\left(a_{0}+b_{0} x_{1}\right)\right)
\end{aligned}
$$

Since $\left(u_{0}^{\prime}, w_{0}\right)=1$, there exists a representation

$$
w_{0}=w_{1} w_{2} w_{3}
$$

and non-zero integers $y_{1}, y_{2}, y_{3}$ such that

$$
a_{0}+b_{0} x_{i}=w_{i} y_{i}, \quad i=1,2,3 .
$$

In particular,

$$
u_{0}^{\prime} y_{1} y_{2} y_{3}=w_{1} w_{2} y_{1} y_{2}+w_{2} w_{3} y_{2} y_{3}+w_{3} w_{1} y_{3} y_{1}
$$

We can assume that

$$
w_{1} \geq w_{2} \geq w_{3} \geq 1 .
$$

By the bound for the divisor function, the representation (17) implies that there are at most $N^{o(1)}$ possible values for $w_{1}, w_{2}, w_{3}$. Let us fix one such representation. Having $w_{1}, w_{2}, w_{3}$ fixed, we observe that the condition $\operatorname{gcd}\left(a_{0}, b_{0}\right)=$ 1 and the equality

$$
a_{0}+b_{0} x_{1}=w_{1} y_{1}
$$

imply that $\operatorname{gcd}\left(b_{0}, w_{1}\right)=1$. Hence, (20) uniquely determines $x_{1}\left(\bmod w_{1}\right)$. It then follows that there are at most

$$
N^{1+o(1)} w_{1}^{-1}+1
$$

possible values for $x_{1}$. Then we retrieve $x_{2}, x_{3}$ and get the bound

$$
J<N^{1+o(1)} w_{1}^{-1}+N^{o(1)} .
$$


Next, from (19) and $w_{1} \geq w_{2} \geq w_{3} \geq 1$ we have

$$
\left|u_{0}^{\prime}\right| \min \left\{\left|y_{1}\right|,\left|y_{2}\right|,\left|y_{3}\right|\right\} \leq 3 w_{1}^{2} \text {. }
$$

Thus,

$$
\min \left\{\left|y_{1}\right|,\left|y_{2}\right|,\left|y_{3}\right|\right\} \leq 3 w_{1}^{2} .
$$

Hence, we can determine one of $y_{1}, y_{2}, y_{3}$ with $O\left(w_{1}^{2}\right)$ possibilities. Consequently, by (18) we determine one of $x_{1}, x_{2}, x_{3}$ and thus we get

$$
J<w_{1}^{2} N^{o(1)} .
$$

Comparing this with (21), we conclude $J<N^{2 / 3+o(1)}$.

\section{Congruences}

In what follows, $N$ is a large parameter, $N<p$. We start with the following result from [12] which is based on the idea of Heath-Brown [20].

Lemma 10. Let $\lambda \not \equiv 0(\bmod p)$. Then the number $J$ of solutions of the congruence

$$
x y \equiv \lambda \quad(\bmod p), \quad L+1 \leq x, y \leq L+N
$$

satisfies

$$
J<\frac{N^{3 / 2+o(1)}}{p^{1 / 2}}+N^{o(1)} .
$$

In particular, if $N<p^{1 / 3}$, then one has $J<N^{o(1)}$.

Corollary 4. Let $\lambda \not \equiv 0(\bmod p)$. Then the number $J$ of solutions of the congruence

$$
\frac{1}{x}+\frac{1}{y} \equiv \lambda \quad(\bmod p), \quad L+1 \leq x, y \leq L+N,
$$

satisfies

$$
J<\frac{N^{3 / 2+o(1)}}{p^{1 / 2}}+N^{o(1)} .
$$

In particular, if $N<p^{1 / 3}$, then one has $J<N^{o(1)}$. 
Proof. Indeed, we have

$$
\left(x-\lambda^{-1}\right)\left(y-\lambda^{-1}\right) \equiv \lambda^{-2} \quad(\bmod p)
$$

and the claim follows from Lemma 10.

The following result has been proved in [12] (see also [8] for the extension of [12] to higher dimensional case).

Lemma 11. Let $\lambda \not \equiv 0(\bmod p)$ and $N<p^{1 / 8}$. Then the number $J$ of solutions of the congruence

$$
x y z \equiv \lambda \quad(\bmod p), \quad L+1 \leq x, y \leq L+N,
$$

satisfies

$$
J<N^{o(1)}
$$

The following lemma follows from the work of Ayyad, Cochrane and Zheng [1] (and from Lemma 10 when $\left|I_{1}\right|\left|I_{2}\right|$ is very small). In fact, we shall only apply this lemma when one of the intervals starts from the origin, the result which had previously been established by Friedlander and Iwaniec [16].

Lemma 12. Let $I_{1}, I_{2}$ be two intervals in $\mathbb{F}_{p}^{*}$ with

$$
\left|I_{1}\right|\left|I_{2}\right|<p
$$

Then the number of solutions of the congruence

$$
x y=z t, \quad(x, z) \in I_{1} \times I_{1}, \quad(y, t) \in I_{2} \times I_{2},
$$

is not greater than $\left(\left|I_{1}\right|\left|I_{2}\right|\right)^{1+o(1)}$.

The following lemma will be used in the proof of Theorems 2 and 3. It is given with explicit constants to make the statement more transparent, the reader should not take them seriously.

Lemma 13. Let $I=\{a+1, \ldots, a+N\}$ and

$$
\lambda \not \equiv 0 \quad(\bmod p), \quad \lambda \notin\left\{x^{-1} \quad(\bmod p): \quad x \in I\right\} .
$$

Assume that

$$
|I|=N<0.1 p^{1 / 18} J^{2 / 9}
$$


where $J$ is the number of solutions of the congruence

$$
x^{-1}+y^{-1}+z^{-1} \equiv \lambda \quad(\bmod p), \quad x, y, z \in I .
$$

Let also $J>N^{\varepsilon}$ for some fixed small constant $\varepsilon>0$ and let $N$ be sufficiently large. Then there exist integers $\Delta_{4}^{\prime}, \Delta_{4}^{\prime \prime}, \Delta_{3}$ with

$$
\left|\Delta_{4}^{\prime}\right|<10^{5} N^{4} / J, \quad\left|\Delta_{4}{ }^{\prime \prime}\right|<10^{5} N^{4} / J, \quad\left|\Delta_{3}\right|<10^{5} N^{3} / J
$$

such that

$$
a \equiv \frac{\Delta_{4}^{\prime}}{\Delta_{3}} \quad(\bmod p) ; \quad \lambda^{-1} \equiv \frac{\Delta_{4}^{\prime \prime}}{\Delta_{3}} \quad(\bmod p)
$$

Proof. It follows that $J$ is the number of solutions of the congruence

$$
\begin{aligned}
\lambda(a+x) & (a+y)(a+z) \\
& \equiv(a+x)(a+y)+(a+y)(a+z)+(a+z)(a+x) \quad(\bmod p)
\end{aligned}
$$

in positive integers $x, y, z \leq N$ with $(a+x)(a+y)(a+z) \not \equiv 0(\bmod p)$.

Note that by Corollary 4 we have $J<N^{1+o(1)}$ so that $N<p^{1 / 13}$. We rewrite the congruence in the form

$$
\begin{aligned}
& x y z+\left(a-\lambda^{-1}\right)(x y+y z+z x)+ \\
& \quad\left(a^{2}-2 a \lambda^{-1}\right)(x+y+z)+\left(a^{3}-3 a^{2} \lambda^{-1}\right) \equiv 0 \quad(\bmod p) .
\end{aligned}
$$

We fix one solution $\left(x_{0}, y_{0}, z_{0}\right)$ and get

$$
\begin{aligned}
& \left(a^{2}-2 a \lambda^{-1}\right)\left(x+y+z-A_{0}\right) \\
& \quad+\left(a-\lambda^{-1}\right)\left(x y+y z+z x-B_{0}\right)+\left(x y z-C_{0}\right) \equiv 0 \quad(\bmod p) .
\end{aligned}
$$

where

$$
A_{0}=x_{0}+y_{0}+z_{0}, \quad B_{0}=x_{0} y_{0}+y_{0} z_{0}+z_{0} x_{0}, \quad C_{0}=x_{0} y_{0} z_{0}
$$

We use some ideas from [9]. Define the lattice

$$
\Gamma=\left\{(u, v, w) \in \mathbb{Z}^{3}:\left(a^{2}-2 a \lambda^{-1}\right) u+\left(a-\lambda^{-1}\right) v+w \equiv 0 \quad(\bmod p)\right\}
$$

and the body

$$
D=\left\{(u, v, w) \in \mathbb{R}^{3}:|u| \leq 3 N,|v| \leq 3 N^{2},|w| \leq N^{3}\right\} .
$$


Since any given vector

$$
\left(x+y+z-A_{0}, x y+y z+z x-B_{0}, x y z-C_{0}\right)
$$

defines the values of $x, y, z$ with at most 6 possibilities, we have

$$
|D \cap \Gamma| \geq J / 6 .
$$

Therefore, by Corollary 3 , the successive minimas $\lambda_{i}=\lambda_{i}(D, \Gamma), i=1,2,3$, satisfy the inequality

$$
\prod_{i=1}^{3} \min \left\{1, \lambda_{i}\right\}<1000 J^{-1}
$$

In particular, we have $\lambda_{1} \leq 1$. By the definition of $\lambda_{i}$, there are linearly independent vectors

$$
\left(u_{i}, v_{i}, w_{i}\right) \in \lambda_{i} D \cap \Gamma, \quad i=1,2,3 .
$$

We consider separately the following three cases.

Case 1: $\lambda_{3} \leq 1$. Thus, we have $\lambda_{1} \lambda_{2} \lambda_{3}<1000 J^{-1}$. We consider the determinant

$$
\Delta=\operatorname{det}\left(\begin{array}{lll}
u_{1} & v_{1} & w_{1} \\
u_{2} & v_{2} & w_{2} \\
u_{3} & v_{3} & w_{3}
\end{array}\right) \text {. }
$$

Clearly,

$$
|\Delta|<6 N^{6} \lambda_{1} \lambda_{2} \lambda_{3}<6000 N^{6} / J<p .
$$

Thus, $|\Delta|<p$. On the other hand, from

$$
\left(a^{2}-2 a \lambda^{-1}\right) u_{i}+\left(a-\lambda^{-1}\right) v_{i}+w_{i} \equiv 0 \quad(\bmod p), \quad i=1,2,3,
$$

we conclude that $\Delta$ is divisible by $p$. Therefore, $\Delta=0$, which contradicts the linear independence of the vectors $\left(u_{i}, v_{i}, w_{i}\right), i=1,2,3$. Thus, this case is impossible.

Case 2: $\lambda_{1} \leq 1, \lambda_{2}>1$. Since $\lambda_{2}>1$ we see that

$$
\left(x+y+z-A_{0}, x y+y z+z x-B_{0}, x y z-C_{0}\right)
$$

and $\left(u_{1}, v_{1}, w_{1}\right)$ are linearly dependent. Therefore, one of the two conditions hold: 
(i) $x+y+z-A_{0}=0$;

(ii) $\left\{\begin{array}{l}x y z=\frac{w_{1}}{u_{1}}(x+y+z)+C_{0}-\frac{w_{1}}{u_{1}} A_{0}, \\ x y+y z+z x=\frac{v_{1}}{u_{1}}(x+y+z)+B_{0}-\frac{v_{1}}{u_{1}} A_{0},\end{array}\right.$

If a solution $(x, y, z)$ satisfy (i), then our congruence (22) can be written in the form

$$
\left(x+a-\frac{1}{\lambda}\right)\left(y+a-\frac{1}{\lambda}\right)\left(z+a-\frac{1}{\lambda}\right) \equiv \lambda^{\prime} \quad(\bmod p) .
$$

Since $\lambda \notin I^{-1}(\bmod p)$, we have $\lambda^{\prime} \not \equiv 0(\bmod p)$. Hence, by Lemma 11 the solutions counted in (i) contributes to our $J$ at most the quantity $N^{o(1)}$.

If a solution $(x, y, z)$ satisfy (ii), then Lemma 7 and the bound for the divisor function implies that one of the variables $x, y, z$ is determined with at most $N^{o(1)}$ possibilities. Therefore, by Corollary 4 we get that the solutions counted on (ii) contributes to our $J$ also at most the quantity $N^{o(1)}$.

Thus, we get $J<N^{o(1)}$ contradicting our assumption that $J>N^{\varepsilon}$. Therefore, Case 2 is impossible.

Case 3: $\lambda_{1} \leq 1, \lambda_{2} \leq 1, \lambda_{3}>1$.

Thus, $\lambda_{1} \lambda_{2}<1000 J^{-1}$. Next,

$$
\left(\begin{array}{ll}
u_{1} & v_{1} \\
u_{2} & v_{2}
\end{array}\right)\left(\begin{array}{c}
a^{2}-2 a \lambda^{-1} \\
a-\lambda^{-1}
\end{array}\right) \equiv\left(\begin{array}{c}
-w_{1} \\
-w_{2}
\end{array}\right) \quad(\bmod p) .
$$

Let

$$
\Delta_{3}=\operatorname{det}\left(\begin{array}{ll}
u_{1} & v_{1} \\
u_{2} & v_{2}
\end{array}\right), \Delta_{5}=\operatorname{det}\left(\begin{array}{ll}
-w_{1} & v_{1} \\
-w_{2} & v_{2}
\end{array}\right), \Delta_{4}=\operatorname{det}\left(\begin{array}{ll}
u_{1} & -w_{1} \\
u_{2} & -w_{2}
\end{array}\right) .
$$

We have

$$
\left|\Delta_{3}\right|<2000 N^{3} / J, \quad\left|\Delta_{5}\right|<2000 N^{5} / J, \quad\left|\Delta_{4}\right|<2000 N^{4} / J .
$$

We observe that

$$
\Delta_{3} \not \equiv 0 \quad(\bmod p) .
$$

Indeed, assuming the contrary, from the congruence (24) we get

$$
\Delta_{3} \equiv \Delta_{5} \equiv \Delta_{4} \equiv 0 \quad(\bmod p)
$$


Taking into account (25), this implies that

$$
\Delta_{3}=\Delta_{5}=\Delta_{4}=0 \text {. }
$$

It then follows that the rank of the matrix

$$
\left(\begin{array}{lll}
u_{1} & v_{1} & w_{1} \\
u_{2} & v_{2} & w_{2}
\end{array}\right)
$$

is strictly less than 2 , which contradicts the linear independence of the vectors $\left(u_{i}, v_{i}, w_{i}\right), i=1,2$. Thus, we have (26). Hence,

$$
a^{2}-2 a \lambda^{-1} \equiv \frac{\Delta_{5}}{\Delta_{3}} \quad(\bmod p), \quad a-\lambda^{-1} \equiv \frac{\Delta_{4}}{\Delta_{3}} \quad(\bmod p) .
$$

Using (22), we also have

$$
a^{3}-3 a^{2} \lambda^{-1} \equiv \frac{\Delta_{6}}{\Delta_{3}} \quad(\bmod p)
$$

for some integer $\Delta_{6}$ with

$$
\left|\Delta_{6}\right|<6000 N^{6} / J
$$

We have

$$
\lambda^{-2} \equiv \frac{\Delta_{4}^{2}}{\Delta_{3}^{2}}-\frac{\Delta_{5}}{\Delta_{3}} \equiv \frac{\Delta_{4}^{2}-\Delta_{5} \Delta_{3}}{\Delta_{3}^{2}} \quad(\bmod p) .
$$

Furthermore, substituting $\lambda^{-1} \equiv a-\left(\Delta_{4} / \Delta_{3}\right)$ in the other two equations we get

$$
\begin{array}{cc}
\Delta_{3} a^{2}-2 \Delta_{4} a+\Delta_{5} \equiv 0 & (\bmod p) \\
2 \Delta_{3} a^{3}-3 \Delta_{4} a^{2}+\Delta_{6} \equiv 0 & (\bmod p) .
\end{array}
$$

It follows that

$$
\Delta_{4} a^{2}-2 \Delta_{5} a+\Delta_{6} \equiv 0 \quad(\bmod p) .
$$

Consider the polynomials

$$
P(Z)=\Delta_{4} Z^{2}-2 \Delta_{5} Z+\Delta_{6}, \quad Q(Z)=\Delta_{3} Z^{2}-2 \Delta_{4} Z+\Delta_{5}
$$

Since

$$
P(a) \equiv Q(a) \equiv 0 \quad(\bmod p)
$$


we have

$$
\operatorname{Res}(P, Q) \equiv 0 \quad(\bmod p)
$$

On the other hand

$$
|\operatorname{Res}(P, Q)|<10^{18} N^{18} / J^{4}<p .
$$

Thus,

$$
\operatorname{Res}(P, Q)=0 .
$$

It then follows that the polynomials $P(Z)$ and $Q(Z)$ have a common root. If $Q(Z)$ is irreducible in $\mathbb{Q}[\mathbb{Z}]$, then $Q(Z)$ and $P(Z)$ are linearly dependent, implying that

$$
\Delta_{4}^{2}=\Delta_{5} \Delta_{3} .
$$

In view of $(27)$ this is impossible. Hence, $Q(Z)$ is irreducible in $\mathbb{Q}[\mathbb{Z}]$, and therefore its discriminant is a square of an integer. Thus,

$$
\Delta_{4}^{2}-\Delta_{5} \Delta_{3}=m^{2}, \quad m \in \mathbb{Z}_{+} .
$$

It then follows that $m<10^{4} N^{4} / J$. Furthermore,

$$
\lambda^{-2} \equiv \frac{m^{2}}{\Delta_{3}^{2}} \quad(\bmod p) .
$$

Hence,

$$
\lambda^{-1} \equiv \frac{\Delta_{4}^{\prime}}{\Delta_{3}} \quad(\bmod p), \quad\left|\Delta_{4}^{\prime}\right|=m<10^{4} N^{4} / J .
$$

Consequently

$$
a \equiv \frac{\Delta_{4}}{\Delta_{3}}+\lambda^{-1} \equiv \frac{\Delta_{4}{ }^{\prime \prime}}{\Delta_{3}} \quad(\bmod p), \quad\left|\Delta_{4}{ }^{\prime \prime}\right|<10^{5} N^{4} / J .
$$

\section{Proof of Theorems 1-5}

\subsection{Proof of Theorem 1}

Let $I=[a+1, a+N]$. We first consider the case $N<p^{\frac{k+1}{2 k}}$. Thus, we are aiming to prove that in this case one has the bound

$$
J_{2 k}<N^{2 k^{2} /(k+1)+o(1)} .
$$


Put

$$
V=\left[N^{(k-1) /(k+1)}\right] ; \quad Y=\left[N^{2 /(k+1)}\right] ; \quad I_{1}=[a+1, a+2 N] .
$$

First we define an appropriate subset $\mathcal{V} \in[0.5 \mathrm{~V}, \mathrm{~V}]$. For a given $v \in[0.5 \mathrm{~V}, \mathrm{~V}]$ define the function $\eta_{v}: I_{1} \rightarrow \mathbb{Z}_{+}$by

$$
\eta_{v}\left(u^{\prime}\right)=\left|\left\{\left(u_{1}^{\prime}, v_{1}\right) \in I_{1} \times[0.5 V, V] ; \quad u^{\prime} v_{1} \equiv u_{1}^{\prime} v \quad(\bmod p)\right\}\right| .
$$

By Lemma 12,

$$
\sum_{u^{\prime} \in I_{1}} \sum_{v \in[0.5 V, V]} \eta_{v}\left(u^{\prime}\right)<N^{1+o(1)} V
$$

Therefore, there is a subset $\mathcal{V} \in[0.5 V, V]$ with $|\mathcal{V}| \sim V$ such that

$$
\sum_{u^{\prime} \in I_{1}} \eta_{v}\left(u^{\prime}\right)<N^{1+o(1)} \quad \text { for any } \quad v \in \mathcal{V}
$$

For any fixed integers $y_{i} \in \mathcal{Y}=[0.5 Y, Y]$ and $v \in \mathcal{V}$ the quantity $J_{2 k}$ does not exceed the number of solutions of the congruence

$$
\frac{1}{u_{1}^{\prime}-v y_{1}}+\ldots+\frac{1}{u_{k}^{\prime}-v y_{k}} \equiv \frac{1}{u_{k+1}^{\prime}-v y_{k+1}}+\ldots+\frac{1}{u_{2 k}^{\prime}-v y_{2 k}} \quad(\bmod p)
$$

in integers $u_{i}^{\prime} \in I_{1}$. Thus, summing up over $y_{i}$ and $v \in \mathcal{V}$ we get

$$
Y^{2 k} V J_{2 k} \ll \frac{1}{p} \sum_{n=0}^{p-1} \sum_{v \in \mathcal{V}}\left|\sum_{u^{\prime} \in I_{1}} \sum_{y \in \mathcal{Y}} e_{p}\left(n\left(u^{\prime}-v y\right)^{-1}\right)\right|^{2 k}
$$

For $v \in \mathcal{V}$ and $B$ of the form $B=2^{s}<N$, denote

$$
S_{v, B}=\left\{u^{\prime} \in I_{1} ; \quad 0.5 B \leq \eta_{v}\left(u^{\prime}\right)<B\right\} .
$$

Hence

$$
I_{1}=\bigcup_{B} S_{v, B}
$$

and since $v \in \mathcal{V}$, by (28)

$$
\left|S_{v, B}\right|<\frac{N^{1+o(1)}}{B}
$$


From (29) we clearly have

$$
Y^{2 k} V J_{2 k} \ll \frac{N^{o(1)}}{p} \sum_{B} \sum_{n=0}^{p-1} \sum_{v \in \mathcal{V}}\left(\sum_{u^{\prime} \in S_{v, B}}\left|\sum_{y \in \mathcal{Y}} e_{p}\left(n\left(u^{\prime}-v y\right)^{-1}\right)\right|\right)^{2 k} .
$$

Hence, by the Hölder inequality and (30), we get, for some fixed $B$,

$$
Y^{2 k} V J_{2 k} \ll \frac{N^{o(1)}}{p}\left(\frac{N}{B}\right)^{2 k-1} \sum_{n=0}^{p-1} \sum_{v \in \mathcal{V}} \sum_{u^{\prime} \in S_{v, B}}\left|\sum_{y \in \mathcal{Y}} e_{p}\left(n\left(u^{\prime}-v y\right)^{-1}\right)\right|^{2 k} .
$$

The quantity

$$
\frac{1}{p} \sum_{n=0}^{p-1} \sum_{v \in \mathcal{V}} \sum_{u^{\prime} \in S_{v}, B}\left|\sum_{y \in \mathcal{Y}} e_{p}\left(n\left(u^{\prime}-v y\right)^{-1}\right)\right|^{2 k}
$$

is bounded by the number of solutions of the congruence

$$
\frac{1}{u^{\prime}-v y_{1}}+\ldots+\frac{1}{u^{\prime}-v y_{k}} \equiv \frac{1}{u^{\prime}-v y_{k+1}}+\ldots+\frac{1}{u^{\prime}-v y_{2 k}} \quad(\bmod p)
$$

in variables $v \in \mathcal{V}, u^{\prime} \in S_{v, B}, y \in \mathcal{Y}$. Thus, we obtain the bound

$$
\frac{1}{p} \sum_{n=0}^{p-1} \sum_{v \in \mathcal{V}} \sum_{u^{\prime} \in S_{v, B}}\left|\sum_{y \in \mathcal{Y}} e_{p}\left(n\left(u^{\prime}-v y\right)^{-1}\right)\right|^{2 k} \ll Y^{k} V \frac{N^{1+o(1)}}{B}+Y^{2 k} B .
$$

Hence, from (31) we get

$$
Y^{2 k} V J_{2 k} \ll N^{2 k-1+o(1)} B^{-2 k+1}\left(Y^{k} V \frac{N^{1+o(1)}}{B}+Y^{2 k} B\right)
$$

Thus,

$$
J_{2 k}<N^{2 k+o(1)} Y^{-k}+\frac{N^{2 k-1+o(1)}}{V}<N^{\frac{2 k^{2}}{k+1}+o(1)},
$$

which proves the result in the case $N<p^{\frac{k+1}{2 k}}$.

Let now $N>p^{\frac{k+1}{2 k}}$. We split the interval $I=[a+1, a+N]$ into $K \sim$ $N p^{-\frac{k+1}{2 k}}$ subintervals of length at most $N_{1}=p^{\frac{k+1}{2 k}}$. Thus, for some intervals $I^{(1)}, \ldots, I^{(2 k)}$ of length $N_{1}$ we have the bound

$$
J_{2 k}<K^{2 k} R_{2 k} \ll\left(\frac{N}{p^{\frac{k+1}{2 k}}}\right)^{2 k} R_{2 k}
$$


where $R_{2 k}$ is the number of solutions of the congruence

$$
\frac{1}{x_{1}}+\ldots+\frac{1}{x_{k}} \equiv \frac{1}{x_{k+1}}+\ldots+\frac{1}{x_{2 k}} \quad(\bmod p), \quad x_{i} \in I^{(i)}, i=1, \ldots, 2 k .
$$

Expressing the number of solutions of this congruence in terms of exponential sums, applying the Hölder inequality we get that

$$
R_{2 k} \leq \prod_{i=1}^{2 k}\left(R_{2 k}(i)\right)^{1 / 2 k}
$$

where $R_{2 k}(i)$ is the number of solutions of the congruence

$$
\frac{1}{x_{1}}+\ldots+\frac{1}{x_{k}} \equiv \frac{1}{x_{k+1}}+\ldots+\frac{1}{x_{2 k}} \quad(\bmod p), \quad x_{1}, \ldots, x_{2 k} \in I^{(i)} .
$$

Thus, for some fixed $i=i_{0}$ one has

$$
R_{2 k}<R_{2 k}\left(i_{0}\right)
$$

Since $\left|I^{\left(i_{0}\right)}\right|<N_{1}=p^{\frac{k+1}{2 k}}$, we already know that

$$
R_{2 k}\left(i_{0}\right)<N_{1}^{2 k^{2} /(k+1)+o(1)}=p^{k} N^{o(1)} .
$$

Thus,

$$
J_{2 k}<\left(\frac{N}{p^{\frac{k+1}{2 k}}}\right)^{2 k} p^{k} N^{o(1)}=\frac{N^{2 k+o(1)}}{p},
$$

which concludes the proof of Theorem 1 .

\subsection{Proof of Theorem 2}

Let $I=\{a+1, \ldots, a+N\}$. We can assume that $N$ is large and $J>$ $N^{2 / 3} \log N$, as otherwise there is nothing to prove. The conditions of Lemma 13 are satisfied, so that there exist integers $\Delta_{4}^{\prime}, \Delta_{4}^{\prime \prime}$ and $\Delta_{3}$ with

$$
\left|\Delta_{4}^{\prime}\right|<N^{10 / 3}, \quad\left|\Delta_{4}^{\prime \prime}\right|<N^{10 / 3}, \quad\left|\Delta_{3}\right|<N^{7 / 3}
$$

such that

$$
a \equiv \frac{\Delta_{4}^{\prime}}{\Delta_{3}} \quad(\bmod p) ; \quad \lambda^{-1} \equiv \frac{\Delta_{4}^{\prime \prime}}{\Delta_{3}} \quad(\bmod p) .
$$


Substituting this in

$$
\begin{aligned}
& \lambda(a+x)(a+y)(a+z) \\
& \quad \equiv(a+x)(a+y)+(a+y)(a+z)+(a+z)(a+x) \quad(\bmod p)
\end{aligned}
$$

we obtain

$$
\begin{aligned}
& \left(\Delta_{4}^{\prime}+\Delta_{3} x\right)\left(\Delta_{4}^{\prime}+\Delta_{3} y\right)\left(\Delta_{4}^{\prime}+\Delta_{3} z\right) \equiv \Delta_{4}^{\prime \prime}\left\{\left(\Delta_{4}^{\prime}+\Delta_{3} x\right)\left(\Delta_{4}^{\prime}+\Delta_{3} y\right)\right. \\
& \left.+\left(\Delta_{4}^{\prime}+\Delta_{3} y\right)\left(\Delta_{4}^{\prime}+\Delta_{3} z\right)+\left(\Delta_{4}^{\prime}+\Delta_{3} z\right)\left(\Delta_{4}^{\prime}+\Delta_{3} x\right)\right\} \quad(\bmod p) .
\end{aligned}
$$

The left and the right hand sides are of the order of magnitude $O\left(N^{10}\right)=$ $o(p)$. Thus, the congruence is converted to the equality

$$
\begin{gathered}
\left(\Delta_{4}^{\prime}+\Delta_{3} x\right)\left(\Delta_{4}^{\prime}+\Delta_{3} y\right)\left(\Delta_{4}^{\prime}+\Delta_{3} z\right)=\Delta_{4}^{\prime \prime}\left\{\left(\Delta_{4}^{\prime}+\Delta_{3} x\right)\left(\Delta_{4}^{\prime}+\Delta_{3} y\right)\right. \\
\left.+\left(\Delta_{4}^{\prime}+\Delta_{3} y\right)\left(\Delta_{4}^{\prime}+\Delta_{3} z\right)+\left(\Delta_{4}^{\prime}+\Delta_{3} z\right)\left(\Delta_{4}^{\prime}+\Delta_{3} x\right)\right\}
\end{gathered}
$$

and the claim follows from Lemma 9.

\subsection{Proof of Theorem 3}

Let $I=\{a+1, \ldots, a+N\}$. The statement is equivalent to the claim that for any $\varepsilon>0$ one has the bound

$$
J_{6} \ll N^{3+\varepsilon}
$$

where the implied constant may depend only $\varepsilon$.

Observe that for any $j \in \mathbb{Z}$ there are $u_{j}, v_{j} \in \mathbb{Z}$ such that

$$
\frac{u_{j}}{v_{j}} \equiv j \quad(\bmod p) ; \quad\left|u_{j}\right| \leq p^{1 / 2}, \quad 0<\left|v_{j}\right| \leq p^{1 / 2}
$$

This follows from the fact that among more than $p$ numbers

$$
u+j v, \quad 0 \leq u, v \leq\left[p^{1 / 2}\right]
$$

there are at least two numbers congruent modulo $p$. We also represent $a$ in this form, that is

$$
a \equiv \frac{a_{0}}{b_{0}} \quad(\bmod p) ; \quad\left|a_{0}\right| \leq p^{1 / 2}, \quad 0<\left|b_{0}\right| \leq p^{1 / 2} .
$$


Let $T_{j}$ be the number of solutions of the congruence

$$
\frac{1}{a+x_{1}}+\frac{1}{a+x_{2}}+\frac{1}{a+x_{3}} \equiv j \quad(\bmod p) ; \quad 1 \leq x_{1}, x_{2}, x_{3} \leq N .
$$

We have

$$
J_{6}=\sum_{j=0}^{p-1} T_{j}^{2}
$$

and

$$
\sum_{j=0}^{p-1} T_{j} \leq N^{3}
$$

From Corollary 4 it clearly follows that $T_{j}<N^{1+o(1)}$. Therefore, it follows that the contribution to $J_{6}$ from $j \in\left\{I^{-1} \cup 0\right\}(\bmod p)$ is

$$
\sum_{\substack{0 \leq \leq \leq p-1 \\ j \in I^{-} \cup j 0}} T_{j}^{2} \leq(N+1) \max _{j} T_{J}^{2}<N^{3+o(1)} .
$$

Furthermore, the contribution to $J_{6}$ from those $j$ for which $T_{j}<N^{0.1 \varepsilon}$ is less than

$$
N^{0.1 \varepsilon} \sum_{j=0}^{p-1} T_{j} \leq N^{3+0.1 \varepsilon}
$$

Thus, if we denote by $\Omega$ the set of integers $j$ with

$$
1 \leq j \leq p-1, \quad j \notin I^{-1} \quad(\bmod p), \quad\left|T_{j}\right|>N^{0.1 \varepsilon},
$$

then

$$
J_{6}<N^{3+0.2 \varepsilon}+\sum_{j \in \Omega} T_{j}^{2} .
$$

Now for each $j$ we apply Lemma 13 (where $J$ is substituted by $T_{j}$ and $\lambda$ by $j$. Then there exist numbers $\Delta_{4 j}^{\prime}, \Delta_{4 j}^{\prime \prime}, \Delta_{3 j}$ with

$$
\Delta_{4 j}^{\prime} \ll N^{4}, \quad \Delta_{4 j}{ }^{\prime \prime} \ll N^{4}, \quad \Delta_{3 j} \ll N^{3}
$$

such that

$$
a \equiv \frac{\Delta_{4 j}^{\prime}}{\Delta_{3 j}} \quad(\bmod p) ; \quad j^{-1} \equiv \frac{\Delta_{4 j}^{\prime \prime}}{\Delta_{3 j}} \quad(\bmod p) .
$$


Comparing this with (32) and (33), we see that

$$
a \equiv \frac{a_{0}}{b_{0}} \equiv \frac{\Delta_{4 j}^{\prime}}{\Delta_{3 j}} \quad(\bmod p) ; \quad j^{-1} \equiv \frac{v_{j}}{u_{j}} \equiv \frac{\Delta_{4 j}^{\prime \prime}}{\Delta_{3 j}} \quad(\bmod p) .
$$

Taking into account the inequality conditions on $N, a_{0}, b_{0}, u_{j}, v_{j}$, we see that we have equality

$$
\frac{a_{0}}{b_{0}}=\frac{\Delta_{4 j}^{\prime}}{\Delta_{3 j}} ; \quad \frac{v_{j}}{u_{j}}=\frac{\Delta_{4 j}^{\prime \prime}}{\Delta_{3 j}} .
$$

Now we represent the congruence corresponding to $T_{j}$ in the form

$$
\begin{aligned}
x_{1} x_{2} x_{3} & +\left(a-j^{-1}\right)\left(x_{1} x_{2}+x_{2} x_{3}+x_{3} x_{1}\right) \\
& +\left(a^{2}-2 a j^{-1}\right)\left(x_{1}+x_{2}+x_{3}\right)+\left(a^{3}-3 a^{2} j^{-1}\right) \equiv 0 \quad(\bmod p) .
\end{aligned}
$$

Using (35), we substitute $a$ and $j^{-1}$, implying

$$
\begin{aligned}
x_{1} x_{2} x_{3} & +\left(\frac{\Delta_{4 j}^{\prime}}{\Delta_{3 j}}-\frac{\Delta_{4 j}^{\prime \prime}}{\Delta_{3 j}}\right)\left(x_{1} x_{2}+x_{2} x_{3}+x_{3} x_{1}\right) \\
& +\left(\left(\frac{\Delta_{4 j}^{\prime}}{\Delta_{3 j}}\right)^{2}-2 \frac{\Delta_{4 j}^{\prime}}{\Delta_{3 j}} \cdot \frac{\Delta_{4 j}^{\prime \prime}}{\Delta_{3 j}}\right)\left(x_{1}+x_{2}+x_{3}\right) \\
& +\left(\left(\frac{\Delta_{4 j}^{\prime}}{\Delta_{3 j}}\right)^{3}-3\left(\frac{\Delta_{4 j}^{\prime}}{\Delta_{3 j}}\right)^{2} \cdot \frac{\Delta_{4 j}^{\prime \prime}}{\Delta_{3 j}}\right) \equiv 0 \quad(\bmod p) .
\end{aligned}
$$

After multiplying by $\Delta_{3 j}^{3}$ the left hand side becomes an integer of the size $O\left(N^{12}\right)=o(p)$. Thus, the resulting congruence is converted to the equality, and dividing by $\Delta_{3 j}^{3}$ we get

$$
\begin{aligned}
x_{1} x_{2} x_{3} & +\left(\frac{\Delta_{4 j}^{\prime}}{\Delta_{3 j}}-\frac{\Delta_{4 j}^{\prime \prime}}{\Delta_{3 j}}\right)\left(x_{1} x_{2}+x_{2} x_{3}+x_{3} x_{1}\right) \\
& +\left(\left(\frac{\Delta_{4 j}^{\prime}}{\Delta_{3 j}}\right)^{2}-2 \frac{\Delta_{4 j}^{\prime}}{\Delta_{3 j}} \cdot \frac{\Delta_{4 j}^{\prime \prime}}{\Delta_{3 j}}\right)\left(x_{1}+x_{2}+x_{3}\right) \\
& +\left(\left(\frac{\Delta_{4 j}^{\prime}}{\Delta_{3 j}}\right)^{3}-3\left(\frac{\Delta_{4 j}^{\prime}}{\Delta_{3 j}}\right)^{2} \cdot \frac{\Delta_{4 j}^{\prime \prime}}{\Delta_{3 j}}\right)=0 .
\end{aligned}
$$


We use (36) and write this equality in the form

$$
\begin{aligned}
x_{1} x_{2} x_{3} & +\left(\frac{a_{0}}{b_{0}}-\frac{v_{j}}{u_{j}}\right)\left(x_{1} x_{2}+x_{2} x_{3}+x_{3} x_{1}\right) \\
& +\left(\frac{a_{0}^{2}}{b_{0}^{2}}-2 \frac{a_{0}}{b_{0}} \cdot \frac{v_{j}}{u_{j}}\right)\left(x_{1}+x_{2}+x_{3}\right) \\
& +\left(\frac{a_{0}^{3}}{b_{0}^{3}}-3 \frac{a_{0}^{2}}{b_{0}^{2}} \cdot \frac{v_{j}}{u_{j}}\right)=0 .
\end{aligned}
$$

Consequently we get

$$
\frac{1}{\left(a_{0} / b_{0}\right)+x_{1}}+\frac{1}{\left(a_{0} / b_{0}\right)+x_{2}}+\frac{1}{\left(a_{0} / b_{0}\right)+x_{3}}=\frac{u_{j}}{v_{j}} .
$$

Thus, for $j \in \Omega$ the quantity $T_{j}$ is just the number of solutions of the diophantine equation (37) in positive integers $x_{1}, x_{2}, x_{3} \leq N$. Since $u_{j} / v_{j}$ are pairwise distinct, we get that the quantity $\sum_{j \in \Omega} T_{j}^{2}$ is not greater than the number of solutions of the equation

$$
\begin{aligned}
\frac{1}{\left(a_{0} / b_{0}\right)+x_{1}}+ & \frac{1}{\left(a_{0} / b_{0}\right)+x_{2}}+\frac{1}{\left(a_{0} / b_{0}\right)+x_{3}} \\
& =\frac{1}{\left(a_{0} / b_{0}\right)+x_{4}}+\frac{1}{\left(a_{0} / b_{0}\right)+x_{5}}+\frac{1}{\left(a_{0} / b_{0}\right)+x_{6}}
\end{aligned}
$$

in positive integers $x_{1}, \ldots, x_{6} \leq N$. Therefore, by Lemma 6 we get that

$$
\sum_{j \in \Omega} T_{j}^{2}<N^{3+o(1)}
$$

In view of (34), this completes the proof of our theorem.

\subsection{Proof of Theorem 4}

Let $I=\{a+1, \ldots, a+N\}$. Using standard arguments involving Hölder's inequality (combined with inductive process), it suffices to show that the contribution from the set of solutions with pairwise $x_{1}, \ldots, x_{2 k}$ is $N^{k+o(1)}$. Thus, in what follows, we consider $x_{1}, \ldots, x_{2 k}$ pairwise distinct.

For each solution $\vec{x}=\left(x_{1}, \ldots, x_{2 k}\right)$, we consider the polynomial $P_{\vec{x}}(Z)$ defined as

$$
\prod_{i \neq 1}\left(Z+x_{i}\right)+\ldots+\prod_{i \neq k}\left(Z+x_{i}\right)-\prod_{i \neq k+1}\left(Z+x_{i}\right)-\ldots-\prod_{i \neq 2 k}\left(Z+x_{i}\right) .
$$


Clearly, $\operatorname{deg} P_{\vec{x}}(Z) \leq 2 k-2$. Note also that $P_{\vec{x}}\left(-x_{1}\right) \neq 0$. In particular, $P_{\vec{x}}(Z)$ is not a zero polynomial, and since $P_{\vec{x}}(a) \equiv 0(\bmod p)$, it is not a constant polynomial neither. Clearly, $P_{\vec{x}}(Z)$ has the form

$$
P_{\vec{x}}(Z)=\sum_{i=0}^{2 k-2} a_{i} Z^{2 k-2-i}
$$

where $\left|a_{i}\right| \ll N^{i+1}$. We fix one solution

$$
\left(x_{1}, \ldots, x_{2 k}\right)=\left(c_{1}, \ldots, c_{2 k}\right)
$$

and consider the polynomial $P_{\vec{c}}(Z)$ that corresponds to $\left(c_{1}, \ldots, c_{2 k}\right)$. Since

$$
P_{\vec{x}}(a) \equiv P_{\vec{c}}(a) \equiv 0 \quad(\bmod p),
$$

we get that

$$
\operatorname{Res}\left(P_{\vec{x}}(Z), P_{\vec{c}}(Z)\right) \equiv 0 \quad(\bmod p) .
$$

On the other hand, $P_{\vec{x}}(Z)$ and $P_{\vec{c}}(Z)$ satisfy the condition of Lemma 2 with

$$
\sigma=\theta=1, \quad m=n=2 k-1 .
$$

Hence,

$$
\operatorname{Res}\left(P_{\vec{x}}(Z), P_{\vec{c}}(Z)\right) \ll N^{4 k^{2}-4 k} .
$$

Therefore, assuming $N<p^{1 /\left(4 k^{2}\right)}$, we get

$$
\left|\operatorname{Res}\left(P_{\vec{x}}(Z), P_{\vec{c}}(Z)\right)\right|<p,
$$

whence

$$
\operatorname{Res}\left(P_{\vec{x}}(Z), P_{\vec{c}}(Z)\right)=0 .
$$

It follows that for every solution $\vec{x}=\left(x_{1}, \ldots, x_{2 k}\right)$ the polynomial $P_{\vec{x}}(Z)$ has a common root with $P_{\vec{c}}(Z)$. Since $x_{i}$ are pairwise distinct, the condition $P_{\vec{x}}(\sigma)=0$ implies that $x_{i}+\sigma \neq 0$. Thus, Lemma 6 implies that for every root $\sigma$ of $P_{\vec{c}}(Z)$ the equation $P_{\vec{x}}(\sigma)=0$ has at most $N^{k+o(1)}$ solutions in positive integers $x_{i} \leq N$. The claim now follows. 


\subsection{Proof of Theorems 5 and 6}

First we prove Theorem 5. It suffices to consider the case $k N^{k-1}<p$ as otherwise the statement is trivial. For $\lambda=0,1, \ldots, p-1$ denote

$$
J(\lambda)=\left\{\left(x_{1}, \ldots, x_{k}\right) \in I^{k}: \quad x_{1}^{*}+\ldots+x_{k}^{*} \equiv \lambda \quad(\bmod p)\right\} .
$$

Let

$$
\Omega=\{\lambda \in[1, p-1]: \quad|J(\lambda)| \geq 1\} .
$$

Since $J(0)=0$, we have

$$
J_{2 k}=\sum_{\lambda \in \Omega}|J(\lambda)|^{2}
$$

Consider the lattice

$$
\Gamma_{\lambda}=\left\{(u, v) \in \mathbb{Z}^{2}: \quad \lambda u \equiv v \quad(\bmod p)\right\}
$$

and the body

$$
D=\left\{(u, v) \in \mathbb{R}^{2}: \quad|u| \leq N^{k},|v| \leq k N^{k-1}\right\} .
$$

Denoting by $\mu_{1}, \mu_{2}$ the consecutive minimas of the body $D$ with respect to the lattice $\Gamma_{\lambda}$, by Corollary 3 it follows

$$
\prod_{i=1}^{2} \min \left\{\mu_{i}, 1\right\} \leq \frac{15}{\left|\Gamma_{\lambda} \cap D\right|} .
$$

Observe that for $\left(x_{1}, \ldots, x_{k}\right) \in J(\lambda)$ one has

$$
\lambda x_{1} \ldots x_{k} \equiv x_{2} \ldots x_{k}+\ldots+x_{1} \ldots x_{k-1} \quad(\bmod p),
$$

implying

$$
\left(x_{1} \ldots x_{k}, x_{2} \ldots x_{k}+\ldots+x_{1} \ldots x_{k-1}\right) \in \Gamma_{\lambda} \cap D .
$$

Thus, for $\lambda \in \Omega$ we have $\mu_{1} \leq 1$. We split the set $\Omega$ into two subsets:

$$
\Omega^{\prime}=\left\{\lambda \in \Omega: \quad \mu_{2} \leq 1\right\}, \quad \Omega^{\prime \prime}=\left\{\lambda \in \Omega: \quad \mu_{2}>1\right\}
$$

We have

$$
J_{2 k}=\sum_{\lambda \in \Omega^{\prime}}|J(\lambda)|^{2}+\sum_{\lambda \in \Omega^{\prime \prime}}|J(\lambda)|^{2} .
$$


Case 1: $\lambda \in \Omega^{\prime}$, that is $\mu_{2} \leq 1$. Let $\left(u_{i}, v_{i}\right) \in \mu_{i} D \cap \Gamma_{\lambda}, i=1,2$, be linearly independent. Then

$$
0 \neq \operatorname{det}\left(\begin{array}{ll}
u_{1} & v_{1} \\
u_{2} & v_{2}
\end{array}\right) \equiv 0 \quad(\bmod p)
$$

whence

$$
\left|\operatorname{det}\left(\begin{array}{ll}
u_{1} & v_{1} \\
u_{2} & v_{2}
\end{array}\right)\right| \geq p
$$

Also

$$
\left|\operatorname{det}\left(\begin{array}{ll}
u_{1} & v_{1} \\
u_{2} & v_{2}
\end{array}\right)\right| \leq 2 k \mu_{1} \mu_{2} N^{2 k-1} \leq \frac{30 k N^{2 k-1}}{|\Gamma \cap D|} .
$$

Thus, for $\lambda \in \Omega^{\prime}$, the number $\left|\Gamma_{\lambda} \cap D\right|$ of solutions of the congruence

$$
\lambda u \equiv v \quad(\bmod p)
$$

in integers $u, v$ with $|u| \leq N^{k},|v| \leq k N^{k-1}$ is bounded by

$$
\left|\Gamma_{\lambda} \cap D\right| \leq \frac{30 k N^{2 k-1}}{p} .
$$

Therefore, if we denote by $S(u, v)$ the set of $k$-tuples $\left(x_{1}, \ldots, x_{k}\right)$ of positive integers $x_{1}, \ldots, x_{k} \leq N$ with

$$
x_{1} \ldots x_{k}=u, \quad x_{2} \ldots x_{k}+\ldots+x_{1} \ldots x_{k-1}=v
$$

we get

$$
\sum_{\lambda \in \Omega^{\prime}}|J(\lambda)|^{2}=\sum_{\lambda \in \Omega^{\prime}}\left(\sum_{(u, v) \in \Gamma_{\lambda} \cap D} \sum_{\left(x_{1}, \ldots, x_{k}\right) \in S(u, v)} 1\right)^{2} .
$$

Applying the Cauchy-Schwarz inequality and taking into account (39), we get

$$
\sum_{\lambda \in \Omega^{\prime}}|J(\lambda)|^{2}=\frac{30 k N^{2 k-1}}{p} \sum_{\lambda \in \Omega^{\prime}} \sum_{(u, v) \in \Gamma_{\lambda} \cap D}\left(\sum_{\left(x_{1}, \ldots, x_{k}\right) \in S(u, v)} 1\right)^{2}
$$

The summation on the right hand side is clearly bounded by the number of solutions of the system of equations

$$
\left\{\begin{array}{l}
x_{1} \ldots x_{k}=y_{1} \ldots y_{k} \\
x_{1} \ldots x_{k-1}+\ldots+x_{2} \ldots x_{k}=y_{2} \ldots y_{k}+\ldots+y_{1} \ldots y_{k-1}
\end{array}\right.
$$


in positive integers $x_{i}, y_{j} \leq N$. Hence, by Lemma 4 , it follows that

$$
\sum_{\lambda \in \Omega^{\prime}}|J(\lambda)|^{2}<30 k(2 k)^{80 k^{3}}(\log N)^{4 k^{2}} \frac{N^{3 k-1}}{p} .
$$

Case 2: $\lambda \in \Omega^{\prime \prime}$, that is $\mu_{2}>1$. Then the vectors from $\Gamma \cap D$ are linearly dependent and in particular there is some $\widehat{\lambda} \in \mathbb{Q}$ such that

$$
\widehat{\lambda} x_{1} \ldots x_{k}=x_{2} \ldots x_{k}+\ldots+x_{1} \ldots x_{k-1} \quad \text { for } \quad\left(x_{1}, \ldots, x_{k}\right) \in J(\lambda) .
$$

Thus,

$$
\begin{aligned}
& \sum_{\lambda \in \Omega^{\prime \prime}}|J(\lambda)|^{2} \leq \sum_{\widehat{\lambda} \in \mathbb{Q}} \mid\left\{\left(x_{1}, \ldots, x_{k}\right) \in I^{k}: \frac{1}{x_{1}}+\ldots+\frac{1}{x_{k}}=\left.\widehat{\lambda}\right|^{2}\right. \\
= & \left|\left\{\left(x_{1}, \ldots, x_{2 k}\right) \in[1, N]^{2 k}: \quad \frac{1}{x_{1}}+\ldots+\frac{1}{x_{k}}=\frac{1}{x_{k+1}}+\ldots+\frac{1}{x_{2 k}}\right\}\right| \\
< & (2 k)^{80 k^{3}}(\log N)^{4 k^{2}} N^{k} .
\end{aligned}
$$

Inserting this and (40) into (38), we obtain

$$
J_{2 k}<(2 k)^{90 k^{3}}(\log N)^{4 k^{2}}\left(\frac{N^{2 k-1}}{p}+1\right) N^{k}
$$

which concludes the proof of Theorem 5 .

The proof of Theorem 6 follows the same line with the only difference that instead of Lemma 4 one should apply the bound

$$
\begin{gathered}
\left|\left\{\left(x_{1}, \ldots, x_{2 k}\right) \in([1, N] \cap \mathcal{P})^{2 k}: \frac{1}{x_{1}}+\ldots+\frac{1}{x_{k}}=\frac{1}{x_{k+1}}+\ldots+\frac{1}{x_{2 k}}\right\}\right| \\
<(2 k)^{k}\left(\frac{N}{\log N}\right)^{k} .
\end{gathered}
$$

\section{Proof of Theorems $7-13$}

\subsection{Proof of Theorem 7}

It suffices to deal with the case $\left|I_{1}\right|=\left[p^{1 / 18}\right],\left|I_{2}\right|=\left[p^{5 / 12+\varepsilon}\right]$ and $\varepsilon<0.1$. Let

$$
W_{2}=\sum_{x_{1} \in I_{1}} \sum_{x_{2} \in I_{2}} \alpha_{1}\left(x_{1}\right) \alpha_{2}\left(x_{2}\right) e_{p}\left(a x_{1}^{*} x_{2}^{*}\right) .
$$


We take $k=[1 / \varepsilon]$ and apply the Hölder inequality;

$$
\begin{aligned}
\left|W_{2}\right|^{k} & \leq\left|I_{1}\right|^{k-1} \sum_{x_{1} \in I_{1}}\left|\sum_{x_{2} \in I_{2}} \alpha_{2}\left(x_{2}\right) e_{p}\left(a x_{1}^{*} x_{2}^{*}\right)\right|^{k} \\
& =\left|I_{1}\right|^{k-1} \sum_{x_{1} \in I_{1}}\left|\sum_{y_{1}, \ldots, y_{k} \in I_{2}} \alpha_{2}\left(y_{1}\right) \ldots \alpha_{2}\left(y_{k}\right) e_{p}\left(a x_{1}^{*}\left(y_{1}^{*}+\ldots+y_{k}^{*}\right)\right)\right| \\
& =\left|I_{1}\right|^{k-1} \sum_{x_{1} \in I_{1}} \theta\left(x_{1}\right)\left(\sum_{y_{1}, \ldots, y_{k} \in I_{2}} \alpha_{2}\left(y_{1}\right) \ldots \alpha_{2}\left(y_{k}\right) e_{p}\left(a x_{1}^{*}\left(y_{1}^{*}+\ldots+y_{k}^{*}\right)\right)\right) \\
& \leq\left|I_{1}\right|^{k-1} \sum_{y_{1}, \ldots, y_{k} \in I_{2}}\left|\sum_{x_{1} \in I_{1}} \theta\left(x_{1}\right) e_{p}\left(a x_{1}^{*}\left(y_{1}^{*}+\ldots+y_{k}^{*}\right)\right)\right|,
\end{aligned}
$$

where $\theta(x)$ some complex numbers with $|\theta(x)| \leq 1$. We again apply the Hölder inequality and obtain

$$
\begin{aligned}
\left|W_{2}\right|^{3 k} & \leq\left|I_{1}\right|^{3 k-3}\left|I_{2}\right|^{2 k} \sum_{y_{1}, \ldots, y_{k} \in I_{2}}\left|\sum_{x_{1} \in I_{1}} \theta\left(x_{1}\right) e_{p}\left(a x_{1}^{*}\left(y_{1}^{*}+\ldots+y_{k}^{*}\right)\right)\right|^{3} \\
& =\left|I_{1}\right|^{3 k-3}\left|I_{2}\right|^{2 k} \sum_{\lambda=0}^{p-1} T(\lambda)\left|\sum_{x_{1} \in I_{1}} \theta\left(x_{1}\right) e_{p}\left(a x_{1}^{*} \lambda\right)\right|^{3}
\end{aligned}
$$

where $T(\lambda)$ is the number of solutions of the congruence

$$
y_{1}^{*}+\ldots+y_{k}^{*} \equiv \lambda \quad(\bmod p), \quad y_{i} \in I_{2} .
$$

We apply now the Cauchy-Schwarz inequality and get

$$
\left|W_{2}\right|^{6 k} \leq\left|I_{1}\right|^{6 k-6}\left|I_{2}\right|^{4 k}\left(\sum_{\lambda=0}^{p-1} T(\lambda)^{2}\right) \sum_{\lambda=0}^{p-1}\left|\sum_{x_{1} \in I_{1}} \theta\left(x_{1}\right) e_{p}\left(a x_{1}^{*} \lambda\right)\right|^{6} .
$$

By Theorem 1, we have

$$
\sum_{\lambda=0}^{p-1} T(\lambda)^{2}<\left|I_{2}\right|^{2 k-2+1 /(k+1)+o(1)}<\left|I_{2}\right|^{2 k-2+\varepsilon} .
$$

Furthermore,

$$
\sum_{\lambda=0}^{p-1}\left|\sum_{x_{1} \in I_{1}} \theta\left(x_{1}\right) e_{p}\left(a x_{1}^{*} \lambda\right)\right|^{6} \leq p J_{6}
$$


where $J_{6}$ is the number of solutions of the congruence

$$
y_{1}^{*}+y_{2}^{*}+y_{3}^{*} \equiv y_{4}^{*}+y_{5}^{*}+y_{6}^{*} \quad(\bmod p), \quad y_{i} \in I_{1} \text {. }
$$

By Theorem 3, we have $J_{6}=\left|I_{1}\right|^{3+o(1)}$. Thus,

$$
\left|W_{2}\right|^{6 k} \leq p\left|I_{1}\right|^{6 k-3+o(1)}\left|I_{2}\right|^{6 k-2+\varepsilon} .
$$

Since $p \ll\left|I_{1}\right|^{3}\left|I_{2}\right|^{2} p^{-2 \varepsilon}$, the result follows.

\subsection{Proof of Theorems 8, 9, 10}

Let

$$
S=\sum_{x_{1} \in I_{1}} \sum_{x_{2} \in I_{2}} \alpha_{1}\left(x_{1}\right) \alpha_{2}\left(x_{2}\right) e_{p}\left(a x_{1}^{*} x_{2}^{*}\right) .
$$

Then by Hölder's inequality

$$
|S|^{k_{2}} \leq N_{1}^{k_{2}-1} \sum_{x_{1} \in I_{1}}\left|\sum_{x_{2} \in I_{2}} \alpha_{2}\left(x_{2}\right) e_{p}\left(a x_{1}^{*} x_{2}^{*}\right)\right|^{k_{2}} \text {. }
$$

Thus, for some $\sigma\left(x_{1}\right) \in \mathbb{C},\left|\sigma\left(x_{1}\right)\right|=1$,

$$
|S|^{k_{2}} \leq N_{1}^{k_{2}-1} \sum_{y_{1}, \ldots, y_{k_{2}} \in I_{2}} \mid \sum_{x_{1} \in I_{1}} \sigma\left(x_{1}\right) e_{p}\left(a x_{1}^{*}\left(y_{1}^{*}+\ldots+y_{k_{2}}^{*}\right) \mid .\right.
$$

Again by Hölder's inequality,

$$
|S|^{k_{1} k_{2}} \leq N_{1}^{k_{1} k_{2}-k_{1}} N_{2}^{k_{1} k_{2}-k_{2}} \sum_{\lambda=0}^{p-1} J_{k_{2}}\left(\lambda ; N_{2}\right) \mid \sum_{x_{1} \in I_{1}} \sigma\left(x_{1}\right) e_{p}\left(\left.a x_{1}^{*} \lambda\right|^{k_{1}}\right.
$$

where $J_{k}(\lambda ; N)$ is the number of solutions of the congruence

$$
x_{1}^{*}+\ldots+x_{k}^{*} \equiv \lambda \quad(\bmod p), \quad x_{i} \in[1, N] .
$$

Then applying the Cauchy-Schwarz inequality and using

$$
\sum_{\lambda=0}^{p-1} J_{k_{2}}\left(\lambda ; N_{2}\right)^{2}=J_{2 k_{2}}\left(N_{2}\right), \quad \sum_{\lambda=0}^{p-1} \mid \sum_{x_{1} \in I_{1}} \sigma\left(x_{1}\right) e_{p}\left(\left.a x_{1}^{*} \lambda\right|^{2 k_{1}} \leq p J_{2 k_{1}}\left(N_{1}\right) .\right.
$$


we get

$$
|S|^{2 k_{1} k_{2}} \leq p N_{1}^{2 k_{1} k_{2}-2 k_{1}} N_{2}^{2 k_{1} k_{2}-2 k_{2}} J_{2 k_{1}}\left(N_{1}\right) J_{2 k_{2}}\left(N_{2}\right) .
$$

Applying Theorem 5, we obtain

$$
\begin{aligned}
|S|^{2 k_{1} k_{2}} & \leq\left(2 k_{1}\right)^{90 k_{1}^{3}}\left(2 k_{2}\right)^{90 k_{2}^{3}}\left(\log N_{1}\right)^{4 k_{1}^{2}}\left(\log N_{2}\right)^{4 k_{2}^{2}} \times \\
& \times N_{1}^{2 k_{1} k_{2}} N_{2}^{2 k_{1} k_{2}}\left(\frac{N_{1}^{k_{1}-1}}{p^{1 / 2}}+\frac{p^{1 / 2}}{N^{k_{1}}}\right)\left(\frac{N_{2}^{k_{2}-1}}{p^{1 / 2}}+\frac{p^{1 / 2}}{N^{k_{2}}}\right) .
\end{aligned}
$$

Thus,

$$
\begin{aligned}
|S| & <\left(2 k_{1}\right)^{45 k_{1}^{2} / k_{2}}\left(2 k_{2}\right)^{45 k_{2}^{2} / k_{1}}(\log p)^{2\left(\frac{k_{1}}{k_{2}}+\frac{k_{2}}{k_{1}}\right)} \times \\
& \times\left(\frac{N_{1}^{k_{1}-1}}{p^{1 / 2}}+\frac{p^{1 / 2}}{N^{k_{1}}}\right)^{1 /\left(2 k_{1} k_{2}\right)}\left(\frac{N_{2}^{k_{2}-1}}{p^{1 / 2}}+\frac{p^{1 / 2}}{N^{k_{2}}}\right)^{1 /\left(2 k_{1} k_{2}\right)} N_{1} N_{2},
\end{aligned}
$$

which finishes the proof of Theorem 8 .

To prove Theorem 9 , we note that if $1 \leq N<p$, then as a consequence of Corollary 4, the number of solutions of the congruence

$$
\frac{1}{x_{1}}+\frac{1}{x_{2}} \equiv \frac{1}{x_{3}}+\frac{1}{x_{4}} \quad(\bmod p), \quad L+1 \leq x_{1}, x_{2}, x_{3}, x_{4} \leq L+N
$$

is bounded by $N^{2+o(1)}\left(N^{3 / 2} p^{-1 / 2}+1\right)$. Following the proof of Theorem 8 with $k_{1}=k_{2}=2$ and applying this bound with $[L+1, L+N]=I_{i}$, we derive Theorem 9.

To prove Theorem 10 we use (41), where in this case $J_{2 k_{i}}\left(N_{i}\right)$ is the number of solutions of the congruence

$$
x_{1}^{-1}+\ldots+x_{k}^{-1}=x_{k+1}^{-1}+\ldots+x_{2 k}^{-1}, \quad\left(x_{1}, \ldots, x_{2 k}\right) \in I_{i}^{2 k} .
$$

Since

$$
N_{1}<p^{\frac{k_{1}+1}{2 k_{1}}}, \quad N_{2}<p^{\frac{k_{2}+1}{2 k_{2}}}
$$

by Theorem 1 we have

$$
J_{2 k_{1}}\left(N_{1}\right)<N_{1}^{\frac{2 k_{1}^{2}}{k_{1}+1}}, \quad J_{2 k_{2}}\left(N_{2}\right)<N_{2}^{\frac{2 k_{2}^{2}}{k_{2}+1}} .
$$

Incorporating this in (41), the result follows. 


\subsection{Proof of Theorem 11}

Denote

$$
W_{n}=\left|\sum_{x_{1} \in I_{1}} \ldots \sum_{x_{n} \in I_{n}} \alpha_{1}\left(x_{1}\right) \ldots \alpha_{n}\left(x_{n}\right) e_{p}\left(a x_{1}^{*} \ldots x_{n}^{*}\right)\right| .
$$

Applying $n$ times the Hölder inequality and using that for $|\alpha(v)| \leq 1$ one has

$$
\sum_{u}\left|\sum_{v} \alpha(v) e_{p}(a u v)\right|^{6} \leq \sum_{v_{1}, \ldots, v_{6}}\left|\sum_{u} e_{p}\left(a\left(v_{1}+v_{2}+v_{3}-v_{4}-v_{5}-v_{6}\right) u\right)\right|,
$$

it follows that

$$
\begin{aligned}
W_{n}^{6^{n}} & \leq N^{n 6^{n}-6 n} \times \\
& \times \sum_{x_{11}, \ldots, x_{16} \in I_{1}} \ldots \sum_{x_{n 1}, \ldots x_{n 6} \in I_{n}} e_{p}\left(a\left(x_{11}^{*}+\ldots-x_{16}^{*}\right) \ldots\left(x_{n 1}^{*}+\ldots-x_{n 6}^{*}\right)\right) .
\end{aligned}
$$

We can fix $x_{j 4}, x_{j 5}, x_{j 6}$ such that for some integers $c_{j}$

$$
W_{n}^{6^{n}} \leq N^{n 6^{n}-3 n}\left|\sum_{x_{11}, x_{12}, x_{13} \in I_{1}} \cdots \sum_{x_{n 1}, x_{n 2}, x_{n 3} \in I_{n}}\{\ldots\}\right|
$$

where in the brackets $\{\ldots\}$ we have the expression

$$
e_{p}\left(a\left(x_{11}^{*}+x_{12}^{*}+x_{13}^{*}-c_{1}\right) \ldots\left(x_{n 1}^{*}+x_{n 2}^{*}+x_{n 3}^{*}-c_{n}\right)\right) .
$$

Let $T_{j}(\lambda), j=1, \ldots, n$, be the number of solutions of the congruence

$$
x_{1}^{*}+x_{2}^{*}+x_{3}^{*}-c_{j} \equiv \lambda \quad(\bmod p), \quad x_{1}, x_{2}, x_{3} \in I_{j} .
$$

Then we get

$$
W_{n}^{6^{n}} \leq N^{n 6^{n}-3 n}\left|\sum_{\lambda_{1}=0}^{p-1} \ldots \sum_{\lambda_{n}=0}^{p-1} T_{1}\left(\lambda_{1}\right) \ldots T_{n}\left(\lambda_{n}\right) e_{p}\left(a \lambda_{1} \ldots \lambda_{n}\right)\right| .
$$

Now we observe that

$$
\sum_{\lambda=0}^{p-1} \frac{T_{j}(\lambda)}{N^{3}} \leq 1
$$

and also by Theorem 3 we have

$$
\sum_{\lambda}\left(\frac{T_{j}(\lambda)}{N^{3}}\right)^{2}<N^{-3+o(1)}
$$


Furthermore,

$$
\prod_{j=1}^{n}\left(\sum_{\lambda}\left(\frac{T_{j}(\lambda)}{N^{3}}\right)^{2}\right)^{1 / 2}<N^{-3 n / 2+o(1)}<p^{-1 / 2-\delta}
$$

for some $\delta=\delta(\varepsilon, n)>0$. Thus, we can apply Lemma 1 with

$$
\gamma_{j}(x)=\frac{T_{j}(x)}{N^{3}}
$$

This implies that

$$
\left|\sum_{\lambda_{n}=0}^{p-1} T_{1}\left(\lambda_{1}\right) \ldots T_{n}\left(\lambda_{n}\right) e_{p}\left(a \lambda_{1} \ldots \lambda_{n}\right)\right|<N^{3 n} p^{-\delta^{\prime}} .
$$

Inserting this into (42), we conclude the proof.

\subsection{Proof of Theorem 12}

Let $c \leq 1 / 4$ be the constant that satisfies Theorem 4 and take $C=9 c^{-2}$. In particular, we can assume that $n>3 c^{-1}$. Clearly, we can also assume that $N=\left[p^{9 /\left(c n^{2}\right)}\right]$. For $k=[c n / 3]$ we have

$$
p^{c / k^{2}} \geq p^{9 /\left(c n^{2}\right)}>N .
$$

Thus, for every $j$ the number of solutions of the congruence

$$
y_{1}^{*}+\ldots+y_{k}^{*} \equiv y_{k+1}^{*}+\ldots+y_{2 k}^{*} \quad(\bmod p), \quad y_{1}, \ldots, y_{2 k} \in I_{j},
$$

is bounded by $N^{k+o(1)}$. Letting

$$
W_{n}=\left|\sum_{x_{1} \in I_{1}} \ldots \sum_{x_{n} \in I_{n}} \alpha_{1}\left(x_{1}\right) \ldots \alpha_{n}\left(x_{n}\right) e_{p}\left(a x_{1}^{*} \ldots x_{n}^{*}\right)\right|,
$$

we have

$$
W_{n}^{(2 k)^{n}} \leq N^{n(2 k)^{n}-2 k n} \sum_{x_{11}, \ldots, x_{(2 k) 1} \in I_{1}} \ldots \sum_{x_{1 n}, \ldots, x_{(2 k) n} \in I_{n}} e_{p}(a\{\ldots\})
$$

where $\{\ldots\}$ denotes

$$
\prod_{j=1}^{n}\left(x_{1 j}^{*}+\ldots+x_{k j}^{*}-x_{(k+1) j}^{*}-\ldots-x_{(2 k) j}^{*}\right) .
$$


We can fix $x_{(k+i) j}$ for all $i=1, \ldots, k$ and $j=1, \ldots, n$ such that for some integers $c_{1}, \ldots, c_{n}$ we have

$$
W_{n}^{(2 k)^{n}} \leq N^{n(2 k)^{n}-k n}\left|\sum_{x_{11}, \ldots, x_{k 1} \in I_{1}} \cdots \sum_{x_{1 n}, \ldots, x_{k n} \in I_{n}} e_{p}(a\{\ldots\})\right|
$$

where $\{\ldots\}$ denotes

$$
\left(x_{11}^{*}+\ldots+x_{k 1}^{*}-c_{1}\right) \ldots\left(x_{1 n}^{*}+\ldots+x_{k n}^{*}-c_{n}\right) .
$$

Thus,

$$
W_{n}^{(2 k)^{n}} \leq N^{n(2 k)^{n}-k n}\left|\sum_{\lambda_{1}=0}^{p-1} \ldots \sum_{\lambda_{n}=0}^{p-1} T_{1}\left(\lambda_{1}\right) \ldots T_{n}\left(\lambda_{n}\right) e_{p}\left(a \lambda_{1} \ldots \lambda_{n}\right)\right|,
$$

where $T_{j}\left(\lambda_{j}\right)$ is the number of solutions of the congruence

$$
y_{1}^{*}+\ldots+y_{k}^{*}-c_{j} \equiv \lambda_{j} \quad(\bmod p), \quad\left(y_{1}, \ldots, y_{k}\right) \in I_{j}^{k} .
$$

We have

$$
\sum_{\lambda_{j}=0}^{p-1} \frac{T_{j}\left(\lambda_{j}\right)}{N^{k}} \leq 1
$$

Furthermore, by Theorem 4

$$
\sum_{\lambda_{j}=0}^{p-1}\left(\frac{T_{j}\left(\lambda_{j}\right)}{N^{k}}\right)^{2}<\frac{N^{k+o(1)}}{N^{2 k}}<N^{-k+o(1)}<p^{-\delta}
$$

and

$$
\prod_{j=1}^{n}\left(\sum_{\lambda_{j}=0}^{p-1}\left(\frac{T_{j}\left(\lambda_{j}\right)}{N^{k}}\right)^{2}\right)^{1 / 2}<N^{-k n / 2} p^{o(1)}<p^{-1 / 2-\delta}
$$

for some $\delta=\delta(\varepsilon, n)>0$. Here we used that

$$
N=\left[p^{9 /\left(c n^{2}\right)}\right] ; \quad N^{k n / 2}>N^{c n^{2} / 12} \gg p^{3 / 4} .
$$

Thus, we can apply Lemma 1, leading to

$$
\sum_{\lambda_{1}=0}^{p-1} \ldots \sum_{\lambda_{n}=0}^{p-1} T_{1}\left(\lambda_{1}\right) \ldots T_{n}\left(\lambda_{n}\right) e_{p}\left(a \lambda_{1} \ldots \lambda_{n}\right)<N^{2 k n} p^{-\delta^{\prime}}
$$

for some $\delta^{\prime}=\delta^{\prime}(\varepsilon, n)>0$. Joining this with (43), we conclude the proof of Theorem 12 . 


\subsection{Proof of Theorem 13}

Put $N_{j}=\left|I_{j}\right|$. Removing, if necessary, intervals $I_{j}$ with $N_{j} \leq p^{\varepsilon /(2 n)}$ we can easily reduce the problem to the case when $N_{j}>p^{\varepsilon /(2 n)}$ for all $j$ and $N_{1} \ldots N_{n} \geq p^{1 / 2+\varepsilon}$. Also note that if $N_{j} \geq p^{1 / 2+\varepsilon /(10 n)}$, then the claim follows from Weil's bound for incomplete Kloosterman sums. Thus, we can also assume that $N_{j}<p^{1 / 2+\varepsilon /(10 n)}$ for all $j$. Then those intervals $I_{j}$ for which $N_{j}>p^{1 / 2}$ we refine to subintervals of sizes $\approx p^{1 / 2}$ and thus can assume that $p^{\varepsilon /(2 n)}<N_{j}<p^{1 / 2}$ for all $j$. Again we can refine the intervals in an obvious way and eventually reduce the problem to the case when

$$
p^{1 / 2+\varepsilon}<N_{1} \ldots N_{n}<2 p^{1 / 2+\varepsilon}
$$

and

$$
p^{\varepsilon /(2 n)}<N_{j}<p^{1 / 2}, \quad j=1,2, \ldots, n .
$$

Let

$$
W_{n}=\left|\sum_{x_{1} \in I_{1}} \ldots \sum_{x_{n} \in I_{n}} \alpha_{1}\left(x_{1}\right) \ldots \alpha_{n}\left(x_{n}\right) e_{p}\left(a x_{1}^{*} \ldots x_{n}^{*}\right)\right| .
$$

Taking $k=[2 / \varepsilon]$ and consequently applying Hölder's inequality $n$ times, we get

$$
\begin{aligned}
W_{n}^{(2 k)^{n}} \leq & \left(N_{1} \ldots N_{n}\right)^{(2 k)^{n}-2 k} \times \\
& \sum_{\lambda_{1}=0}^{p-1} \ldots \sum_{\lambda_{n}=0}^{p-1} T_{1}\left(\lambda_{1}\right) \ldots T_{n}\left(\lambda_{n}\right) e_{p}\left(a \lambda_{1} \ldots \lambda_{n}\right),
\end{aligned}
$$

where $T_{j}\left(\lambda_{j}\right)$ is the number of solutions of the congruence

$$
\left(y_{1}^{*}+\ldots+y_{k}^{*}\right)-\left(y_{k+1}^{*}+\ldots+y_{2 k}^{*}\right) \equiv \lambda_{j} \quad(\bmod p), \quad\left(y_{1}, \ldots, y_{2 k}\right) \in I_{j}^{2 k} .
$$

Now we observe that

$$
\sum_{\lambda_{j}=0}^{p-1} \frac{T_{j}\left(\lambda_{j}\right)}{N_{j}^{2 k}} \leq 1
$$

Furthermore, by Theorem 1

$$
\sum_{\lambda_{j}=0}^{p-1}\left(\frac{T_{j}\left(\lambda_{j}\right)}{N_{j}^{2 k}}\right)^{2}<\frac{N_{j}^{4 k-2+1 /(2 k+1)+o(1)}}{N_{j}^{4 k}}<N_{j}^{-2+1 /(2 k+1)+o(1)}<p^{-\delta}
$$


and

$$
\prod_{j=1}^{n}\left(\sum_{\lambda_{j}=0}^{p-1}\left(\frac{T_{j}\left(\lambda_{j}\right)}{N_{j}^{2 k}}\right)^{2}\right)^{1 / 2}<\left(N_{1} \ldots N_{n}\right)^{-1} p^{0.5 \varepsilon}<p^{-1 / 2-\delta}
$$

for some $\delta=\delta(\varepsilon, n)>0$. Thus, we can apply Lemma 1, leading to

$$
\sum_{\lambda_{1}=0}^{p-1} \ldots \sum_{\lambda_{n}=0}^{p-1} T_{1}\left(\lambda_{1}\right) \ldots T_{n}\left(\lambda_{n}\right) e_{p}\left(a \lambda_{1} \ldots \lambda_{n}\right)<\left(N_{1} \ldots N_{n}\right)^{2 k} p^{-\delta^{\prime}}
$$

for some $\delta^{\prime}=\delta^{\prime}(\varepsilon, n)>0$. Joining this with (44), we conclude the proof of Theorem 13 .

\section{Proof of Theorem 14}

We need the following consequence of [7, Theorem 7]. Let $\mu, \nu$ be positive probability measures on $\mathbb{R}$ supported on $[-1,1], \alpha, \beta$ complex functions on $\mathbb{R} ;|\alpha|,|\beta| \leq 1$. Let $\xi \in \mathbb{R},|\xi|>1$. Then

$$
\left|\iint \alpha(x) \beta(y) e^{i x y \xi} \mu(d x) \nu(d y)\right| \ll|\xi|^{-1 / 2}\left\|\mu * \varphi_{\delta}\right\|_{2}\left\|\nu * \varphi_{\delta}\right\|_{2},
$$

where $\delta=(100|\xi|)^{-1}$ and

$$
\varphi_{\delta}(t)= \begin{cases}\delta^{-1} & \text { if } t \in\left[-\frac{\delta}{2}, \frac{\delta}{2}\right] \\ 0 & \text { otherwise. }\end{cases}
$$

Note that (45) is very simple, the paper [7] contains also multi-linear versions derived from discretized ring theorem, but we do not need them here.

Let us give a direct proof of (45). For the brevity write $\varphi=\varphi_{\delta}$. Since

$$
\widehat{\varphi}(\lambda)=\frac{2}{\delta \lambda} \sin \frac{\delta \lambda}{2}
$$

we have, for $|\lambda|<(10 \delta)^{-1}=10 \xi$ and $|y| \leq 1$,

$$
\frac{1}{2}<\widehat{\varphi}(\lambda) \leq 1, \quad\left|\frac{\beta(y)}{\widehat{\varphi}(\xi y)}\right| \leq 2 .
$$

Write for $x, y \in[-1,1]$

$$
e^{i \xi x y}=\frac{1}{\widehat{\varphi}(\xi y)} \int \varphi(s-x) e^{i \xi s y} d s .
$$


Hence,

$$
\begin{aligned}
& \left|\iint \alpha(x) \beta(y) e^{i x y \xi} \mu(d x) \nu(d y)\right| \leq \\
& \iint\left|\int \frac{\beta(y)}{\widehat{\varphi}(\xi y)} e^{i \xi s y} \nu(d y)\right| \varphi(s-x) \mu(d x) d s= \\
& \quad \int \widehat{\nu_{1}}(\xi s)(\mu * \varphi)(s) d s,
\end{aligned}
$$

where

$$
\frac{d \nu_{1}}{d \nu}=\frac{\beta(y)}{\widehat{\varphi}(\xi y)}, \quad \text { hence } \quad\left|\nu_{1}\right| \leq 2 \nu .
$$

Since $\operatorname{supp}(\mu * \varphi) \subset[-2,2]$, it follows from (46) that

$$
\left|\iint \alpha(x) \beta(y) e^{i x y \xi} \mu(d x) \nu(d y)\right| \leq\|\mu * \phi\|_{2}\left\|\widehat{\nu_{1}}(\xi \cdot)\right\|_{L^{2}[-2,2]} .
$$

Next, for $|s| \leq 2$

$$
\left|\widehat{\nu_{1}}(\xi s)\right| \leq 2\left|\widehat{\nu_{1}}(\xi s) \widehat{\varphi}(\xi s)\right|=2 \mid\left(\nu_{1} * \varphi\right) \widehat{(\xi s) \mid .}
$$

Therefore

$$
\begin{aligned}
\left\|\widehat{\nu_{1}}(\xi \cdot)\right\|_{L}^{2}[-2,2] & \leq 2|\xi|^{-1 / 2}\left\|\left(\nu_{1} * \varphi\right) \widehat{~}\right\|_{2} \\
& =2|\xi|^{-1 / 2}(2 \pi)^{1 / 2}\left\|\nu_{1} * \varphi\right\|_{2} \\
& \leq 4(2 \pi)^{1 / 2}|\xi|^{-1 / 2}\|\nu * \varphi\|_{2}
\end{aligned}
$$

and inequality (45) follows.

Let $e(z)=e^{i z}$. Denoting

$$
S=\sum_{\substack{n_{1} \sim N_{1} \\ n_{2} \sim N_{2}}} e\left(\frac{1}{n_{1}} \frac{1}{n_{2}} \xi\right)
$$

we estimate

$$
\begin{aligned}
|S|^{k_{1} k_{2}} & \leq N_{1}^{k_{1} k_{2}-k_{1}} N_{2}^{k_{1} k_{2}-k_{2}} \times \\
& \times \sum_{\substack{n_{11}, \ldots, n_{1 k_{1}} \sim N_{1} \\
n_{21}, \ldots, n_{2 k_{2}} \sim N_{2}}} \alpha_{\overline{n_{1}}} \beta_{\overline{n_{2}}} e\left(\xi\left(\frac{1}{n_{11}}+\ldots+\frac{1}{n_{1 k_{1}}}\right)\left(\frac{1}{n_{21}}+\ldots+\frac{1}{n_{2 k_{2}}}\right)\right),
\end{aligned}
$$


where $\overline{n_{i}}=\left(n_{i 1}, \ldots, n_{i k_{i}}\right)$ and

$$
\alpha_{\overline{n_{1}}}=\alpha_{\left(\frac{1}{n_{11}}+\ldots+\frac{1}{n_{1 k_{1}}}\right)}, \quad \beta_{\overline{n_{2}}}=\beta_{\left(\frac{1}{n_{21}}+\ldots+\frac{1}{n_{2 k_{2}}}\right)}
$$

and $\left|\alpha_{\overline{n_{1}}}\right|=\left|\beta_{\overline{n_{2}}}\right|=1$. Thus, for some complex coefficients $\alpha(x)$ and $\beta(y)$ with $|\alpha(x)|=|\beta(y)|=1$ we have

$$
|S|^{k_{1} k_{2}} \leq\left(N_{1} N_{2}\right)^{k_{1} k_{2}}\left|\iint \alpha(x) \beta(y) e\left(\frac{\xi}{N_{1} N_{2}} x y\right) \mu(d x) \nu(d y)\right|
$$

where $\mu$ is obtained as normalized image measure on $\mathbb{R}$ under the map

$$
\left\{n_{i} \sim N_{1}\right\}^{k_{1}} \rightarrow \mathbb{R}: \quad\left(n_{11}, \ldots, n_{1 k_{1}}\right) \rightarrow N_{1}\left(\frac{1}{n_{11}}+\ldots+\frac{1}{n_{1 k_{1}}}\right)
$$

and similarly for $\nu$.

Set $\delta=\frac{N_{1} N_{2}}{100|\xi|}$. It follows from (45) that

$$
|S|^{k_{1} k_{2}} \leq\left(N_{1} N_{2}\right)^{k_{1} k_{2}} \delta^{1 / 2}\left\|\mu * \varphi_{\delta}\right\|_{2}\left\|\nu * \varphi_{\delta}\right\|_{2} .
$$

Next we estimate $\left\|\mu * \varphi_{\delta}\right\|_{2}$. Note that if $I_{j}$ is a partition of $\mathbb{R}$ in $\delta$-intervals, then

$$
\begin{aligned}
& \sum_{j} \mid\left(n_{1}, \ldots, n_{k}\right) ; n_{i} \sim N \text { and } \frac{N}{n_{1}}+\ldots+\frac{N}{n_{k}} \in I_{j} \mid \leq \\
& \mid\left(n_{1}, \ldots, n_{2 k}\right) ; n_{i} \sim N \text { and }\left|\frac{1}{n_{1}}+\ldots+\frac{1}{n_{k}}-\frac{1}{n_{k+1}}-\ldots-\frac{1}{n_{2 k}}\right|<\frac{\delta}{N} \mid .
\end{aligned}
$$

Thus, it follows that

$$
\left\|\mu * \varphi_{\delta}\right\|_{2}^{2} \sim N_{1}^{-2 k_{1}} \delta^{-1} T\left(N_{1}\right)
$$

where

$T(N)=\mid\left(n_{1}, \ldots, n_{2 k}\right) ; n_{i} \sim N$ and $\left|\frac{1}{n_{1}}+\ldots+\frac{1}{n_{k}}-\frac{1}{n_{k+1}}-\ldots-\frac{1}{n_{2 k}}\right|<\frac{\delta}{N} \mid$.

Let us prove that

$$
T(N)<c(k)(\log N)^{4 k^{2}} N^{k}\left(1+\delta N^{2 k-1}\right) .
$$


For $\lambda \in \mathbb{Q}$, denote $J(\lambda)$ the number of solutions of representations of $\lambda$ as

$$
\lambda=\frac{1}{n_{1}}+\ldots+\frac{1}{n_{k}}, \quad n_{i} \sim N .
$$

By Lemma 4,

$$
\sum_{\lambda} J(\lambda)^{2} \leq c(k) N^{k}(\log N)^{4 k^{2}} .
$$

Also note that different $\lambda$ 's are at least $\sim N^{-2 k}$ separated. Hence an interval $I \subset \mathbb{R}$ of size $\delta / N$ contains at most $1+N^{2 k-1} \delta$ elements of the form (49). Therefore,

$$
\begin{aligned}
T(N) & \leq \sum_{n_{1}, \ldots, n_{k} \sim N} J\left(\frac{1}{n_{1}}+\ldots+\frac{1}{n_{k}}\right)\left(1+N^{2 k-1} \delta\right) \\
& \leq\left(1+N^{2 k-1} \delta\right) \sum_{\lambda} J(\lambda)^{2}<c(k) N^{k}(\log N)^{4 k^{2}}\left(1+N^{2 k-1} \delta\right),
\end{aligned}
$$

which establishes the required bound for $T(N)$.

Thus, from (48) we get

$$
\left\|\mu * \varphi_{\delta}\right\|_{2} \leq c\left(k_{1}\right)\left(\log N_{1}\right)^{2 k_{1}} \delta^{-1 / 2} N_{1}^{-k_{1} / 2}\left(1+\delta N_{1}^{2 k_{1}-1}\right)^{1 / 2} .
$$

Similarly,

$$
\left\|\nu * \varphi_{\delta}\right\|_{2} \leq c\left(k_{2}\right)\left(\log N_{2}\right)^{2 k_{2}} \delta^{-1 / 2} N_{2}^{-k_{2} / 2}\left(1+\delta N_{2}^{2 k_{2}-1}\right)^{1 / 2} .
$$

Inserting these bounds into (47), we get

$$
\begin{aligned}
|S|^{k_{1} k_{2}} & \leq c\left(k_{1}\right) c\left(k_{2}\right)\left(\log N_{1}\right)^{2 k_{1}}\left(\log N_{2}\right)^{2 k_{2}}\left(N_{1} N_{2}\right)^{k_{1} k_{2}} \times \\
& \times\left(\delta^{-1 / 2} N_{1}^{-k_{1}}+\delta^{1 / 2} N_{1}^{k_{1}-1}\right)^{1 / 2}\left(\delta^{-1 / 2} N_{2}^{-k_{2}}+\delta^{1 / 2} N_{2}^{k_{2}-1}\right)^{1 / 2} .
\end{aligned}
$$

Recalling that $\delta=\frac{N_{1} N_{2}}{100|\xi|}$ we conclude the proof.

\section{Some applications}

In this section we apply Theorem 14 to prove Theorem 15 on $\pi(x)-\pi(x-y)$, and apply trilinear exponential sum bounds to prove Theorem 16 on a linear Kloosterman sums and Theorem 17 on Brun-Titchmarsh theorem. 


\subsection{Proof of Theorem 15}

In view of Huxley's result [21] we can assume that $y<x^{7 / 12+}$. The function

$$
\frac{2(1-\theta)}{12\left(\theta^{-1}+1\right)\left(\theta^{-1}+0.5\right)+1-\theta}
$$

increases in $\theta \in[0,7 / 12]$, so we can assume that $y=x^{\theta}$. Going over the argument of $[18, \mathrm{p} .269],(13.56)$ gives a bound on $R(M, N)$ of the form

$$
\frac{H y}{M N}\left|\sum_{\substack{m \sim M \\ n \sim N}} \alpha_{m} \beta_{n} e\left(\frac{h u}{m n}\right)\right|
$$

with $u \sim x$ and $1 \leq h \leq H=M N y^{-1} x^{\varepsilon}$. Here $M, N$ may be chosen arbitrarily with

$$
M N=D>y
$$

(see [18, Theorem 12.21]) and we need to ensure that

$$
R(M, N)<y x^{-\varepsilon} .
$$

We may then state an upper bound

$$
\pi(x)-\pi(x-y)<\frac{2 y}{\log D} .
$$

Thus,

$$
\frac{H y}{M N}\left|\sum_{\substack{m \sim M \\ n \sim N}} \alpha_{m} \beta_{n} e\left(\frac{h u}{m n}\right)\right| \leq x^{\varepsilon}\left|\sum_{\substack{m \sim M \\ n \sim N}} \alpha_{m} \beta_{n} e\left(\frac{\xi}{m n}\right)\right|
$$

where

$$
D<x \leq \xi<\frac{D}{y} x^{1+\varepsilon}
$$

Take $k$ satisfying

$$
k-\frac{1}{2}<\frac{1}{\theta}<k+\frac{1}{2}
$$

and define $M$ by

$$
\frac{x}{D}=M^{2 k-1}
$$

Let

$$
N=\frac{D}{M}
$$


and choose $l$ such that

$$
N^{2(l-1)} \leq \frac{\xi}{D}<N^{2 l}
$$

Hence,

$$
\log N=\log D-\frac{\log \frac{x}{D}}{2 k-1} \geq \log y-\frac{\log \frac{x}{y}}{2 k-1}=\left(\theta-\frac{1-\theta}{2 k-1}\right) \log x>\frac{\theta}{2} \log x
$$

and $l \leq \theta^{-1}+1$. Bounding (50) by Theorem 14 gives

$$
\begin{aligned}
& x^{\varepsilon} D\left(\frac{\xi}{D} M^{-2 k}+\frac{D}{\xi} M^{2(k-1)}\right)^{1 /(4 k l)} \leq \\
& x^{\varepsilon} D\left(\frac{D x^{\varepsilon}}{y} M^{-1}+M^{-1}\right)^{1 /(4 k l)}< \\
& x^{2 \varepsilon}\left(\frac{D}{y}\right)^{5 / 4}\left(\frac{x}{D}\right)^{-1 /(4 k(2 k-1) l)} y< \\
& x^{2 \varepsilon}\left(\frac{D}{y}\right)^{3 / 2} x^{-\frac{(1-\theta) \theta}{8\left(\theta^{-1}+1\right)\left(\theta^{-1}+0.5\right)}} y .
\end{aligned}
$$

Hence we may take

$$
D=y^{1+\frac{1-\theta}{12\left(\theta^{-1}+1\right)\left(\theta^{-1}+0.5\right)}-\varepsilon^{\prime}}
$$

implying Theorem 15.

\subsection{Proof of Theorem 16}

Denote $\varepsilon=\log N / \log p$. As a consequence of the Weil bound on incomplete Kloosterman sums, we can assume that $\varepsilon<4 / 7$. Let

$$
\mathcal{G}=\left\{x<N: \quad p_{1} \geq N^{\alpha}, p_{3} \geq N^{\beta}, p_{1} p_{2} p_{3}<N^{1-\beta}\right\},
$$

where $p_{1} \geq p_{2} \geq p_{3}$ are the largest prime factors of $x$ and

$$
0.1>\alpha>\beta>\frac{1}{\log N}
$$

are parameters to specify. Letting $0.1>\beta_{1}>\beta$ be another parameter, we observe that

$$
\sum_{\substack{x<N \\ p_{1} p_{2}>N^{1-\beta_{1}}}} 1 \leq \sum_{y<N^{\beta_{1}}} \sum_{p_{1} p_{2} \leq N / y} 1 \leq \sum_{y<N^{\beta_{1}}} \sum_{p_{2} \leq N} \frac{3 N}{p_{2} y \log N} \leq 4 \beta_{1}(\log \log N) N .
$$


Similarly,

$$
\sum_{\substack{x<N \\ p_{1} p_{2} p_{3} \geq N^{1-\beta}}} 1<\sum_{y \leq N^{\beta}} \sum_{p_{2} p_{3}<N} \frac{4 N}{y p_{2} p_{3} \log N}<5 \beta(\log \log N)^{2} N .
$$

We also note that the number of positive integers not exceeding $N$ and consisting on products of at most two prime numbers is less than

$$
\frac{2 N \log \log N}{\log N}<2 \beta N \log \log N \text {. }
$$

Hence, we have

$$
\begin{aligned}
N-|\mathcal{G}| \leq & \frac{2 N \log \log N}{\log N}+ \\
& +\sum_{\substack{x<N \\
p_{1}<N^{\alpha}}} 1+\sum_{\substack{x<N \\
p_{1} p_{2}>N^{1-\beta_{1}}}} 1+\sum_{\substack{x<N \\
p_{1} p_{2} \leq N^{1-\beta_{1}} \\
p_{3}<N^{\beta}}} 1+\sum_{\substack{x<N \\
p_{1} p_{2} p_{3} \geq N^{1-\beta}}} 1 \\
& \leq \frac{2 N \log \log N}{\log N}+\Psi\left(N, N^{\alpha}\right)+4 \beta_{1} N \log \log N \\
& +\sum_{p_{1} p_{2}<N^{1-\beta_{1}}} \Psi\left(\frac{N}{p_{1} p_{2}}, N^{\beta}\right)+5 \beta N(\log \log N)^{2} .
\end{aligned}
$$

Here $\Psi(x, y)$, as usual, denotes the number of positive integers $\leq x$ having no prime divisors $>y$. By the classical result of de Bruin [10] if $y>(\log x)^{1+\delta}$, where $\delta>0$ is a fixed constant, then

$$
\Psi(x, y) \leq x u^{-u(1+o(1))} \quad \text { as } \quad u=\frac{\log x}{\log y} \rightarrow \infty .
$$

Thus, taking

$$
\alpha=\frac{1}{\log \log p}, \quad \beta_{1}=\beta \log \log p, \quad \frac{2 \log \log N}{\log N}<\beta<\frac{1}{(\log \log p)^{3}},
$$

we have

$$
\begin{aligned}
N-|\mathcal{G}| & <\alpha^{\frac{1}{2 \alpha}} N+\sum_{p_{1} p_{2}<N^{1-\beta_{1}}} \frac{N}{p_{1} p_{2}}\left(\frac{\beta}{\beta_{1}}\right)^{\frac{\beta_{1}}{2 \beta}}+11 \beta N(\log \log p)^{2} \\
& <\left(\alpha^{\frac{1}{2 \alpha}}+(\log \log N)^{2}\left(\frac{\beta}{\beta_{1}}\right)^{\frac{\beta_{1}}{2 \beta}}+11 \beta(\log \log p)^{2}\right) N \\
& <12 \beta(\log \log p)^{2} N .
\end{aligned}
$$


Therefore

$$
\left|\sum_{x<N} e_{p}\left(a x^{*}\right)\right| \leq 12 \beta(\log \log p)^{2} N+\left|\sum_{x \in \mathcal{G}} e_{p}\left(a x^{*}\right)\right| .
$$

We can further assume that

$$
\varepsilon \beta>\frac{1}{\sqrt{\log p}} .
$$

The sum $\sum_{x \in \mathcal{G}} e_{p}\left(a x^{*}\right)$ may be bounded by

$$
\sum_{p_{1}} \sum_{p_{2}} \sum_{p_{3}}\left|\sum_{y} e_{p}\left(a p_{1}^{*} p_{2}^{*} p_{3}^{*} y^{*}\right)\right|
$$

where the summations are taken over primes $p_{1}, p_{2}, p_{3}$ and integers $y$ such that

$$
p_{1} \geq p_{2} \geq p_{3} ; \quad p_{1} \geq N^{\alpha} ; \quad p_{3} \geq N^{\beta} ; \quad p_{1} p_{2} p_{3} \leq N^{1-\beta}
$$

and

$$
y<\frac{N}{p_{1} p_{2} p_{3}} ; \quad P(y) \leq p_{3} .
$$

Note that if $t$ and $T$ are such that

$$
\left(1-\frac{c}{\log p}\right) p_{3}<t<p_{3}, \quad\left(1-\frac{c}{\log p}\right) \frac{N}{p_{1} p_{2} p_{3}}<T<\frac{N}{p_{1} p_{2} p_{3}},
$$

where $c>0$ is any constant, then we can substitute the condition on $y$ with

$$
P(y) \leq t ; \quad y<T
$$

by changing the sum (51) with an additional term of size at most

$$
\frac{N(\log \log p)^{O(1)}}{\log p}
$$

Thus, for any $t$ and $T$ satisfying (53) we have

$$
\left|\sum_{x \in \mathcal{G}} e_{p}\left(a x^{*}\right)\right|<\frac{N(\log \log p)^{O(1)}}{\log p}+\sum_{p_{1}} \sum_{p_{2}} \sum_{p_{3}}\left|\sum_{y} e_{p}\left(a p_{1}^{*} p_{2}^{*} p_{3}^{*} y^{*}\right)\right|,
$$

where the summations are taken over primes $p_{1}, p_{2}, p_{3}$ and integers $y$ satisfying (52) and (54). 
Now we split the range of variations of primes $p_{1}, p_{2}, p_{3}$ into subintervals of the form $\left[L, L+L(\log p)^{-1}\right]$ and choosing suitable $t$ and $T$ we obtain that for some numbers $M_{1}, M_{2}, M_{3}$ with

$$
M_{1}>0.5 M_{2}>0.2 M_{3}, \quad M_{1}>N^{\alpha}, \quad M_{3} \geq N^{\beta}, \quad M_{1} M_{2} M_{3}<N^{1-\beta}
$$

one has

$$
\begin{aligned}
\left|\sum_{x \in \mathcal{G}} e_{p}\left(a x^{*}\right)\right| & <\frac{N(\log \log p)^{O(1)}}{\log p} \\
& +(\log p)^{10} \sum_{p_{1} \in I_{1} p_{2} \in I_{2} p_{3} \in I_{3}} \sum_{\substack{y \leq M \\
P(y) \leq M_{3}}} e_{p}\left(a p_{1}^{*} p_{2}^{*} p_{3}^{*} y^{*}\right) \mid,
\end{aligned}
$$

where

$$
I_{j}=\left[M_{j}, M_{j}+\frac{M_{j}}{\log p}\right], \quad M=\frac{N}{M_{1} M_{2} M_{3}} \geq N^{\beta} .
$$

Denote

$$
W=\sum_{p_{1} \in I_{1}} \sum_{p_{2} \in I_{2}} \sum_{p_{3} \in I_{3}}\left|\sum_{\substack{y \leq M \\ P(y) \leq M_{3}}} e_{p}\left(a p_{1}^{*} p_{2}^{*} p_{3}^{*} y^{*}\right)\right| .
$$

Applying the Cauchy-Schwarz inequality, we get

$$
W^{2} \leq M_{1} M_{2} M_{3} \sum_{y \leq M} \sum_{z \leq M}\left|\sum_{p_{1} \in I_{1}} \sum_{p_{2} \in I_{2}} \sum_{p_{3} \in I_{3}} e_{p}\left(a p_{1}^{*} p_{2}^{*} p_{3}^{*}\left(y^{*}-z^{*}\right)\right)\right| .
$$

Taking into account the contribution from $y=z$ and then fixing $y \neq z$ we get, for some $b \not \equiv 0(\bmod p)$,

$$
W^{2} \leq \frac{N^{2}}{M}+N M|S|,
$$

where

$$
|S|=\left|\sum_{p_{1} \in I_{1}} \sum_{p_{2} \in I_{2}} \sum_{p_{3} \in I_{3}} e_{p}\left(b p_{1}^{*} p_{2}^{*} p_{3}^{*}\right)\right| .
$$

Define integers $k_{1}, k_{2}, k_{3}$ such that

$$
p^{\frac{1}{2 k_{j}}} \leq M_{j}<p^{\frac{1}{2\left(k_{j}-1\right)}}, \quad j=1,2,3 .
$$


From (55) and the choice of $\alpha$ and $\beta$, it follows, in particular, that

$$
k_{1}<\frac{1}{\varepsilon \alpha}<(\log p)^{1 / 2}, \quad k_{2}, k_{3}<\frac{1}{\varepsilon \beta}<(\log p)^{1 / 2} .
$$

We further take even integers $l_{j} \in\left\{k_{j}, k_{j}+1\right\}, \quad(j=1,2,3)$ and define

$$
\eta_{j}(\lambda)=\left|\left\{\left(x_{1}, \ldots, x_{l_{j}}\right) \in\left(I_{j} \cap \mathcal{P}\right)^{l_{j}}: x_{1}^{*}-x_{2}^{*}+\ldots-x_{l_{j}}^{*} \equiv \lambda \quad(\bmod p)\right\}\right| .
$$

We have

$$
\sum_{\lambda=0}^{p-1} \eta_{j}(\lambda)<M_{j}^{l_{j}}
$$

and, applying Theorem 6,

$$
\begin{aligned}
\sum_{\lambda=0}^{p-1} \eta_{j}(\lambda)^{2} & <M_{j}^{2\left(l_{j}-k_{j}\right)} M_{j}^{k_{j}}\left(2 k_{j}\right)^{k_{j}}\left(\frac{M_{j}^{2 k_{j}-1}}{p}+1\right) \\
& <\left(2 k_{j}\right)^{k_{j}} M_{j}^{2 l_{j}} p^{-1 / 2} .
\end{aligned}
$$

Next we apply consequently the Hölder inequality. We get

$$
|S|^{l_{1}}<\left(M_{2} M_{3}\right)^{l_{1}-1} \sum_{p_{2} \in I_{2}} \sum_{p_{3} \in I_{3}}\left|\sum_{p_{1} \in I_{1}} e_{p}\left(b p_{1}^{*} p_{2}^{*} p_{3}^{*}\right)\right|^{l_{1}}
$$

and since we took $l_{j}$ even, we further get

$$
\begin{aligned}
|S|^{l_{1}} & <\left(M_{2} M_{3}\right)^{l_{1}-1} \sum_{\lambda_{1}=0}^{p-1} \eta_{1}\left(\lambda_{1}\right)\left[\sum_{p_{2} \in I_{2}} \sum_{p_{3} \in I_{3}} e_{p}\left(b \lambda_{1} p_{2}^{*} p_{3}^{*}\right)\right] \\
& <\left(M_{2} M_{3}\right)^{l_{1}-1} \sum_{\lambda_{1}=0}^{p-1} \eta_{1}\left(\lambda_{1}\right) \sum_{p_{3} \in I_{3}}\left|\sum_{p_{2} \in I_{2}} e_{p}\left(b \lambda_{1} p_{2}^{*} p_{3}^{*}\right)\right| .
\end{aligned}
$$

Applying the Hölder inequality and using (59), we obtain

$$
\begin{aligned}
|S|^{l_{1} l_{2}} & <\left(M_{2} M_{3}\right)^{\left(l_{1}-1\right) l_{2}} M_{1}^{\left(l_{2}-1\right) l_{1}} M_{3}^{l_{2}-1} \sum_{\lambda_{1}=0}^{p-1} \eta_{1}\left(\lambda_{1}\right) \sum_{p_{3} \in I_{3}}\left|\sum_{p_{2} \in I_{2}} e_{p}\left(b \lambda_{1} p_{2}^{*} p_{3}^{*}\right)\right|^{l_{2}} \\
& =M_{1}^{l_{1}\left(l_{2}-1\right)} M_{2}^{l_{2}\left(l_{1}-1\right)} M_{3}^{l_{1} l_{2}-1} \sum_{\lambda_{1}=0}^{p-1} \sum_{\lambda_{2}=0}^{p-1} \eta_{1}\left(\lambda_{1}\right) \eta_{2}\left(\lambda_{2}\right)\left|\sum_{p_{3} \in I_{3}} e_{p}\left(b \lambda_{1} \lambda_{2} p_{3}^{*}\right)\right| .
\end{aligned}
$$


We again apply the Hölder inequality and use (59),

$$
\begin{aligned}
|S|^{l_{1} l_{2} l_{3}} & <M_{1}^{l_{1} l_{3}\left(l_{2}-1\right)} M_{2}^{l_{2} l_{3}\left(l_{1}-1\right)} M_{3}^{l_{3}\left(l_{1} l_{2}-1\right)}\left(M_{1}^{l_{1}} M_{2}^{l_{2}}\right)^{l_{3}-1} \times \\
& \times\left|\sum_{\lambda_{1}=0}^{p-1} \sum_{\lambda_{2}=0}^{p-1} \sum_{\lambda_{3}=0}^{p-1} \eta_{1}\left(\lambda_{1}\right) \eta_{2}\left(\lambda_{2}\right) \eta_{3}\left(\lambda_{3}\right) e_{p}\left(b \lambda_{1} \lambda_{2} \lambda_{3}\right)\right| \\
& =M_{1}^{l_{1} l_{2} l_{3}-l_{1}} M_{2}^{l_{1} l_{2} l_{3}-l_{2}} M_{3}^{l_{1} l_{2} l_{3}-l_{3}}\left|S_{1}\right|,
\end{aligned}
$$

where

$$
\left|S_{1}\right|=\left|\sum_{\lambda_{1}=0}^{p-1} \sum_{\lambda_{2}=0}^{p-1} \sum_{\lambda_{3}=0}^{p-1} \eta_{1}\left(\lambda_{1}\right) \eta_{2}\left(\lambda_{2}\right) \eta_{3}\left(\lambda_{3}\right) e_{p}\left(b \lambda_{1} \lambda_{2} \lambda_{3}\right)\right| .
$$

We apply Lemma 1 with $n=3$ and

$$
\gamma_{j}(\lambda)=\frac{\eta_{j}(\lambda)}{M^{l_{j}}}
$$

From (59) it follows that

$$
\left\|\gamma_{i}\right\|_{1}=\sum_{\lambda=0}^{p-1} \frac{\eta_{j}(\lambda)}{M^{l_{j}}} \leq 1
$$

From (60) and (58) it follows that

$$
\left\|\gamma_{i}\right\|_{2}=\left(\sum_{\lambda=0}^{p-1}\left(\frac{\eta_{j}(\lambda)}{M^{l_{j}}}\right)^{2}\right)^{1 / 2}<p^{-1 / 5}
$$

and, in particular,

$$
\prod_{i=1}^{n}\left\|\gamma_{i}\right\|_{2}<p^{-1 / 2-1 / 10}
$$

Thus, Lemma 1 applies and leads to

$$
\left|S_{1}\right|<M_{1} M_{2} M_{3} p^{-c}
$$

for some absolute constant $c>0$. Consequently

$$
|S|<M_{1} M_{2} M_{3} p^{-c_{1} /\left(k_{1} k_{2} k_{3}\right)}<M_{1} M_{2} M_{3} m^{-c_{2} \varepsilon^{3} \alpha \beta^{2}} .
$$

Inserting this into (57) and taking into account that

$$
M>N^{\beta}=p^{\varepsilon \beta}>\exp (\sqrt{\log p}),
$$


we get

$$
W<\frac{N}{\exp (0.5 \sqrt{\log p})}+N m^{-c_{3} \varepsilon^{3} \alpha \beta^{2}} .
$$

Inserting this into (56), we get

$$
\left|\sum_{x \in \mathcal{G}} e_{p}\left(a x^{*}\right)\right|<\frac{N(\log \log p)^{O(1)}}{\log p}+(\log p)^{10} N m^{-c_{3} \varepsilon^{3} \alpha \beta^{2}} .
$$

Therefore

$$
\left|\sum_{x<N} e_{p}\left(a x^{*}\right)\right| \leq \frac{N(\log \log p)^{O(1)}}{\log p}+12 \beta(\log \log p)^{2} N+(\log p)^{10} N m^{-c_{3} \varepsilon^{3} \alpha \beta^{2}} .
$$

Thus, taking

$$
\beta=\frac{C \log \log p}{\varepsilon^{3 / 2}(\log p)^{1 / 2}}
$$

with sufficiently large constant $C$, we obtain

$$
\left|\sum_{x<N} e_{p}\left(a x^{*}\right)\right| \ll \frac{(\log \log p)^{3}}{(\log p)^{1 / 2}} \varepsilon^{-3 / 2} N
$$

which finishes the proof.

\subsection{Proof of Theorem 17}

We only treat the case when $q$ is prime, but the argument generalizes.

We follow [17]. Denote

$$
\begin{aligned}
& \mathcal{A}=\{n \leq x ; n \equiv a \quad(\bmod q)\} \\
& \mathcal{A}_{d}=\{n \in \mathcal{A} ; n \equiv 0 \quad(\bmod d)\} \\
& S(\mathcal{A}, z)=\mid\{n \in \mathcal{A} ;(n, p)=1 \text { for } p<z,(p, q)=1\} \mid, \\
& D=\frac{x^{1-\varepsilon}}{q}
\end{aligned}
$$

Let $D^{1 / 5}<w<y<z=(x / q)^{1 / 3}$, where $w$ and $y$ to specify, and write using Buchstab's identity

$$
\begin{aligned}
S(\mathcal{A}, z) & =S(\mathcal{A}, w)-\sum_{y \leq p<z} S\left(\mathcal{A}_{p}, z\right)-\sum_{w \leq p<y} S\left(\mathcal{A}_{p}, w\right) \\
& +\sum_{w \leq p_{1}<p_{2}<y} S\left(\mathcal{A}_{p_{1} p_{2}}, p_{2}\right) .
\end{aligned}
$$


Applying the basic estimates of the linear sieve on each of the terms in (61) leads to the bound

$$
S(\mathcal{A}, z)<\frac{2 x}{\phi(q) \log D}
$$

see the discussion in [17] and also [18, p.265]. In particular this involves bounding

$$
S\left(\mathcal{A}_{p_{1} p_{2}}, p_{2}\right)<\frac{2 x}{\phi(q) p_{1} p_{2} \log D_{12}} \quad \text { with } \quad D_{12}=\frac{D}{p_{1} p_{2}} .
$$

Here $D_{12}$ is the level of distribution for the sequence $\mathcal{A}_{p_{1} p_{2}}$. The idea from [17] is to improve on (63), in the average over $p_{1}, p_{2}$, by increasing the level $D_{12}$ to some level $D_{12}^{\prime}$.

More precisely, define the reminders

$$
R_{p_{1}, p_{2}, d}=\left|\mathcal{A}_{d p_{1} p_{2}}\right|-\frac{x}{q d p_{1} p_{2}}
$$

that appear as the error terms in the sieving process. The strategy is to bound the collected contribution of $R_{p_{1}, p_{2}, d}$, performing the summation over $p_{1}, p_{2}$.

Subdivide $[w, y]$ in dyadic ranges and estimate

$$
\sum_{\substack{p_{1} \sim P_{1} \\ p_{2} \sim P_{2}}} S\left(\mathcal{A}_{p_{1} p_{2}}, p_{2}\right)
$$

for fixed $P_{1}, P_{2}$. We introduce $D_{12}^{\prime}=D_{12}^{\prime}\left(P_{1}, P_{2}\right)>D_{12}$ such that

$$
\sum_{d<D_{12}^{\prime}}\left|\sum_{\substack{p_{1} \sim P_{1} \\ p_{2} \sim P_{2}}} R_{p_{1}, p_{2}, d}\right|<\frac{x^{1-\varepsilon}}{q} P_{1} P_{2} .
$$

With $D_{12}^{\prime}$ as sieving limit, (63) improves to

$$
S\left(\mathcal{A}_{p_{1} p_{2}}, p_{2}\right)<\frac{2 x}{\phi(q) p_{1} p_{2} \log D_{12}^{\prime}}
$$

on average over $p_{1} \sim P_{1}, p_{2} \sim P_{2}$, provided $p_{2}^{3}>D_{12}^{\prime}$. The gain in (62) becomes then of the order

$$
\begin{aligned}
& \frac{x}{\phi(q)} \sum_{w \leq p_{1}<p_{2}<y} \frac{1}{p_{1} p_{2}}\left(\frac{1}{\log D_{12}}-\frac{1}{\log D_{12}^{\prime}\left(p_{1}, p_{2}\right)}\right) \\
& \leq \frac{x}{\phi(q)} \sum_{w \leq p_{1}<p_{2}<y} \frac{1}{p_{1} p_{2}} \frac{\log \frac{D_{12}^{\prime}\left(p_{1}, p_{2}\right)}{D_{12}}}{\left(\log D_{12}\right)^{2}} .
\end{aligned}
$$


Using the analysis from [17] in order to express (64) as exponential sums, we obtain following bound on the left hand side of (65)

$$
\sum_{d<D_{12}^{\prime}} \sum_{0<|h|<H}\left|\sum_{\substack{p_{1} \sim P_{1} \\ p_{2} \sim P_{2}}}\left(q d p_{1} p_{2}\right)^{-1} \widehat{f}\left(\frac{h}{q d p_{1} p_{2}}\right) e_{q}\left(-a h d^{*} p_{1}^{*} p_{2}^{*}\right)\right|
$$

up to admissible error term. Here

$$
H=q d P_{1} P_{2} x^{2 \varepsilon-1}
$$

and $f \geq 0$ is supported on $x^{1-\varepsilon}<t<x+x^{1-\varepsilon}$ satisfying $\widehat{f}(0)=x$ and $t^{j} f^{(j)}(t) \ll x^{\varepsilon}$ for $j \geq 0$. Standard manipulations permit to express (67) in terms of trilinear sums $\left(D_{12} \leq \widetilde{D}<D_{12}^{\prime}\right)$

$$
\frac{x}{q \widetilde{D} P_{1} P_{2}} H\left|\sum_{\substack{d \in I_{0} \\ p_{1} \in I_{1} \\ p_{2} \in I_{2}}} \alpha_{d} \beta_{p_{1}} \gamma_{p_{2}} e_{q}\left(-a h d^{*} p_{1}^{*} p_{2}^{*}\right)\right|
$$

with $\left|\alpha_{d}\right|,\left|\beta_{p_{1}}\right|,\left|\gamma_{p_{2}}\right| \leq 1$ and

$$
I_{0} \subset[\widetilde{D}, 2 \widetilde{D}], \quad I_{1} \subset\left[P_{1}, 2 P_{1}\right], \quad I_{2} \subset\left[P_{2}, 2 P_{2}\right]
$$

intervals. Note that these intervals always be enlarged to

$$
I_{0}=[\widetilde{D}, 2 \widetilde{D}], \quad I_{1}=\left[P_{1}, 2 P_{1}\right], \quad I_{2}=\left[P_{2}, 2 P_{2}\right] .
$$

Take $\delta=(1-\theta) / 5$ and $w=x^{\delta}, y=x^{2 \delta}$. Note that

$$
\widetilde{D}>D_{12}>\frac{D}{y^{2}}>x^{0.9 \delta} .
$$

Performing the Karatsuba amplification followed by a trilinear estimate gives a saving of a factor $x^{c^{\prime} \delta^{3}}$, hence

$$
\frac{x}{q \widetilde{D} P_{1} P_{2}} H\left|\sum_{\substack{d \in I_{0} \\ p_{1} \in I_{1} \\ p_{2} \in I_{2}}} \alpha_{d} \beta_{p_{1}} \gamma_{p_{2}} e_{q}\left(-a h d^{*} p_{1}^{*} p_{2}^{*}\right)\right|<\frac{x^{1-c^{\prime} \delta^{3}}}{q} H<x^{2 \varepsilon-c^{\prime} \delta^{3}} D_{12}^{\prime} P_{1} P_{2}
$$

which is less than $x^{1-\varepsilon} q^{-1}$ for

$$
D_{12}^{\prime}=\frac{x^{1+c^{\prime \prime} \delta^{3}}}{q P_{1} P_{2}}=D_{12} x^{c^{\prime \prime} \delta^{3}}
$$


The condition $w^{5}>D_{12}^{\prime}$ is satisfied. Thus, returning to (66), we obtain the saving

$$
\frac{x}{\phi(q)} \sum_{x^{\delta}<p_{1}<p_{2}<x^{2 \delta}} \frac{1}{p_{1} p_{2}} \frac{\log \left(x^{c^{\prime \prime} \delta^{3}}\right)}{\delta^{2}(\log x)^{2}} \gg \frac{x \delta}{\phi(q) \log x} \sim \frac{x \delta^{2}}{\phi(q) \log \frac{x}{q}}
$$

and since $\varepsilon$ is arbitrarily small, the result follows.

\section{Comments}

There is an alternative approach to Theorem 17 following the proof of $[18$, Theorem 13.1]. On [18, p.262], there is a bound

$$
R(M, N) \ll x^{\varepsilon}\{(M, N)-\text { bilinear Kloosterman sum }\}+\frac{x^{1-\varepsilon}}{q} .
$$

According to [18, Theorem 12.21], we may consider any factorization $D=$ $M N$. Let $x / q=x^{\delta}$ and take $k$ such that

$$
\frac{1}{2 k-1} \leq \frac{\delta}{2}<\frac{1}{2 k-3}
$$

Let

$$
N=q^{1 /(2 k-1)}, \quad M=\frac{D}{N}
$$

From our Theorem 8 it follows that

$$
\{(M, N) \text { - bilinear Kloosterman sum }\}<M N^{1-c / k^{2}}<D^{1-c \delta^{2}} .
$$

From condition $D^{1-c \delta^{2}}<x^{1-\varepsilon} q^{-1}$ we obtain,

$$
\pi(x ; q, a)<\frac{\left(2-c \delta^{2}\right) x}{\phi(q) \log (x / q)}
$$

As we have mentioned in the introduction one can prove that if $I \subset \mathbb{F}_{p}^{*}$ with $|I|<p^{1 / 2}$, then

$$
\left|I^{-1}+I^{-1}+I^{-1}\right|>|I|^{1.55+o(1)} .
$$


This is better than what one gets from Corollary 1 for $k=3$. Let us prove this bound. We can assume that $|I|=N>p^{1 / 3}$, as Corollary 4 implies a better bound

$$
\left|I^{-1}+I^{-1}\right|>|I|^{2+o(1)} .
$$

Consider the congruence

$$
\frac{1}{x_{1}}+\frac{1}{x_{2}}+\frac{1}{x_{3}} \equiv \frac{1}{x_{4}}+\frac{1}{x_{5}}+\frac{1}{x_{6}} \quad(\bmod p), \quad x_{1}, \ldots, x_{6} \in I .
$$

The number $J_{6}$ of this congruence can be bounded by

$$
J_{6} \leq\left(J_{4} J_{8}\right)^{1 / 2} .
$$

From Theorem (1) it follows that $J_{8}<N^{32 / 5+o(1)}$, and from Corollary 4 we have that

$$
J_{4}<\frac{N^{7 / 2+o(1)}}{p^{1 / 2}} .
$$

Thus,

$$
J_{6}<\frac{N^{99 / 20+o(1)}}{p^{-1 / 4}} .
$$

Using the relationship between the number of solutions of a congruence and the cardinality of the corresponding set, we conclude that

$$
\left|I^{-1}+I^{-1}+I^{-1}\right| \geq N^{21 / 20+o(1)} p^{1 / 4}>N^{1.55+o(1)} .
$$

We remark that the arguments in the proof of Theorem 1 also give the following.

Proposition 1. For any fixed positive integer constants $r$ and $k$ the number $J_{2 k}^{(r)}$ of solutions of the congruence

$$
\frac{1}{x_{1}^{r}}+\ldots+\frac{1}{x_{k}^{r}} \equiv \frac{1}{x_{k+1}^{r}}+\ldots+\frac{1}{x_{2 k}^{r}} \quad(\bmod p), \quad x_{1}, \ldots, x_{2 k} \in I,
$$

satisfies the bound

$$
J_{2 k}^{(r)}<\left(|I|^{2 k^{2} /(k+1)}+\frac{|I|^{2 k}}{p}\right)|I|^{o(1)} .
$$

Thus, in particular we obtain 
Corollary 5. Let $r_{1}, r_{2}, k_{1}, k_{2}$ be fixed positive integer constants, $I_{1}=\left[a_{1}+\right.$ $\left.1, a_{1}+N_{1}\right], I_{2}=\left[a_{2}+1, a_{2}+N_{2}\right]$ and

$$
N_{1}<p^{\frac{k_{1}+1}{2 k_{1}}}, \quad N_{2}<p^{\frac{k_{2}+1}{2 k_{2}}} .
$$

Then for any complex coefficients $\alpha_{1}\left(x_{1}\right), \alpha_{2}\left(x_{2}\right)$ with $\left|\alpha_{i}\left(x_{i}\right)\right| \leq 1$ one has

$$
\begin{aligned}
\max _{(a, p)=1} \mid \sum_{x_{1} \in I_{1}} \sum_{x_{2} \in I_{2}} & \alpha_{1}\left(x_{1}\right) \alpha_{2}\left(x_{2}\right) e_{p}\left(a x_{1}^{-r_{1}} x_{2}^{-r_{2}}\right) \mid< \\
& \left(p^{\frac{1}{2 k_{1} k_{2}}} N_{1}^{-\frac{1}{k_{2}\left(k_{1}+1\right)}} N_{2}^{-\frac{1}{k_{1}\left(k_{2}+1\right)}}\right)\left(N_{1} N_{2}\right)^{1+o(1)} .
\end{aligned}
$$

One may apply the bilinear sums of Corollary 5 together with Vaughan's formula [32] to bound the corresponding sums over primes, similar to those in [5, Theorems A1, A9] and [14, Corollary 1.5].

Corollary 6. Let $r \in \mathbb{Z}_{+}$and $p>N>p^{1 / 2+\varepsilon}$ for some $\varepsilon>0$. Then

$$
\max _{(a, p)=1}\left|\sum_{\substack{x<N \\ x}} e_{p}\left(a x^{-r}\right)\right|<N^{1-\delta}
$$

for some $\delta=\delta(\varepsilon ; r)>0$.

Next we remark that the result from [19] implies the bound

$$
\max _{(a, p)=1}\left|\sum_{\substack{x<p \\ x \text { prime }}} e_{p}\left(a x^{-1}\right)\right|<p^{15 / 16+o(1)} .
$$

Our Corollary 5 leads to

Corollary 7. For any fixed positive integer constant $r$ the following bound holds:

$$
\max _{(a, p)=1}\left|\sum_{\substack{x<p \\ x \text { prime }}} e_{p}\left(a x^{-r}\right)\right|<p^{23 / 24+o(1)} .
$$

Let us prove it. It suffices to establish the bound

$$
\left|\sum_{n \leq p} \Lambda(n) e_{p}\left(a n^{-r}\right)\right|<p^{23 / 24+o(1)}
$$


and then the result follows by partial summation. Here $\Lambda(n)$ is the Mangoldt function.

Below we use $A \lesssim B$ to mean that $A<B p^{o(1)}$. From the Vaughan's identity (see [13, Chapter 24]), we have

$$
\sum_{n \leq p} \Lambda(n) e_{p}\left(a n^{-r}\right) \lesssim W_{1}+W_{2}+W_{3}+W_{4},
$$

where

$$
\begin{aligned}
& W_{1}=\left|\sum_{n \leq U} \Lambda(n) e_{p}\left(a n^{-r}\right)\right| ; \\
& W_{2}=\sum_{n \leq U V}\left|\sum_{m \leq p / n} e_{p}\left(a n^{-r} m^{-r}\right)\right| ; \\
& W_{3}=\sum_{n \leq V}\left|\sum_{m \leq p / n}(\log m) e_{p}\left(a n^{-r} m^{-r}\right)\right| ; \\
& W_{4}=\sum_{U<n \leq p / V}\left|\sum_{V<m \leq p / n} \beta_{m} e_{p}\left(a n^{-r} m^{-r}\right)\right| .
\end{aligned}
$$

Here $U \geq 2, V \geq 2$ are parameters with $U V \leq p$,

$$
\beta_{m}=\sum_{\substack{d \mid m \\ d \leq V}} \mu(d) .
$$

Below we shall also use Weil's bound of the form

$$
\left|\sum_{x=1}^{p-1} e_{p}\left(a x^{-r}+b x\right)\right| \ll p^{1 / 2} .
$$

More precisely, we shall use a consequence of this bound, namely if $I$ is an interval in $\mathbb{F}_{p}$ then

$$
\left|\sum_{x \in I} e_{p}\left(a x^{-r}\right)\right| \lesssim p^{1 / 2}
$$

We take $U=V=p^{1 / 3}$ and estimate $W_{1}$ trivially:

$$
W_{1} \lesssim U=p^{1 / 3} .
$$


To estimate $W_{2}$ we split the range of summation over $n$ into dyadic intervals and get, for some $L \leq p^{2 / 3}$,

$$
W_{2} \lesssim \sum_{L \leq n \leq 2 L}\left|\sum_{m \leq p / n} e_{p}\left(a n^{-r} m^{-r}\right)\right| .
$$

If $L<p^{1 / 3}$ then we apply Weil's bound to the sum over $m$ and get

$$
W_{2} \lesssim L p^{1 / 2} \lesssim p^{5 / 6} \text {. }
$$

If $p^{1 / 3}<L<p^{2 / 3}$ then using the standard smoothing argument we extend the summation over $m$ to $m \leq p / L$ and apply Corollary 5 with $k_{1}=k_{2}=2$ to get

$$
W_{2} \lesssim p^{23 / 24}
$$

To estimate $W_{3}$ we use partial summation (to the sum over $m$ ) and Weil's bound and get

$$
W_{3} \lesssim \sum_{n<p^{1 / 3}} p^{1 / 2} \lesssim p^{5 / 6}
$$

To estimate $W_{4}$, we split the range of summation over $n$ into dyadic intervals and get, for some $p^{1 / 3}<L \leq p^{2 / 3}$,

$$
W_{4} \lesssim \sum_{L \leq n<2 L}\left|\sum_{p^{1 / 3}<m \leq p / n} \beta_{m} e_{p}\left(a n^{-r} m^{-r}\right)\right| .
$$

Applying the smoothing argument to extend the sum over $m$ to $m<p / L$ and using Corollary 5 with $k_{1}=k_{2}=2$, we get

$$
W_{4} \lesssim p^{23 / 24}
$$

and Corollary 7 follows.

\section{References}

[1] A. Ayyad, T. Cochrane and Z. Zheng, 'The congruence $x_{1} x_{2} \equiv x_{3} x_{4}$ $(\bmod p)$, the equation $x_{1} x_{2}=x_{3} x_{4}$ and the mean value of character sums', J. Number Theory, 59 (1996), 398-413.

[2] R. C. Baker, 'Kloosterman sums with prime variable', Acta Arith., (to appear). 
[3] U. Betke, M. Henk and J. M. Wills, 'Successive-minima-type inequalities', Discr. Comput. Geom., 9 (1993), 165-175.

[4] Z. I. Borevich and I. R. Shafarevich, Number Theory, Academic Press, 1966.

[5] J. Bourgain, 'More on sum-product phenomenon in prime fields and its applications', Int. J. Number Theory 1 (2005), 1-32.

[6] J. Bourgain, 'Multilinear exponential sums in prime fields under optimal entropy condition on the sources', Geom. Funct. Anal. 18 (2009), 1477-1502.

[7] J. Bourgain, 'The discretized sum-product and projection theorems', J. Anal. Math. 112 (2010), 193-236.

[8] J. Bourgain, M. Z. Garaev, S. V. Konyagin and I. E. Shparlinski, 'On the hidden shifted power problem', SIAM J. Comp., (to appear).

[9] J. Bourgain, M. Z. Garaev, S. V. Konyagin and I. E. Shparlinski, 'On congruences with products of variables from short intervals and applications', Proc. Steklov Inst. Math. (to appear).

[10] N. G. de Bruijn, 'On the number of positive integers $\leq x$ and free prime factors > y, II', Indag. Math. 28 (1966), 239-247.

[11] D. A. Burgess, 'Partial Gaussian sums', Bull. London Math. Soc., 20 (1988), 589-592.

[12] J. Cilleruelo and M. Z. Garaev, 'Concentration of points on two and three dimensional modular hyperbolas and applications', Geom. Func. Anal., 21 (2011), 892-904.

[13] H. Davenport, "Multiplicative Number Theory". Third edition, revised by H. L. Montgomery. Springer-Verlag, New York, 2000.

[14] E. Fouvry and Ph. Michel 'Sur certaines sommes d'exponentielles sur les nombres premiers', Ann. Sci. cole Norm. Sup. 31 (1998), 93-130.

[15] E. Fouvry and I. E. Shparlinski 'On a ternary quadratic form over primes', Acta Arith. 150 (2011), 285-314. 
[16] J. Friedlander and H. Iwaniec, 'Estimates for character sums', Proc. Amer. Math. Soc., 119 (1993), 365-372.

[17] J. Friedlander and H. Iwaniec, The Brun-Titchmarsh theorem, Analytic number theory (Kyoto, 1996), 85-93, London Math. Soc. Lecture Note Ser., 247, Cambridge Univ. Press, Cambridge, 1997.

[18] J. Friedlander and H. Iwaniec, Opera de Cribro, American Mathematical Society, Colloquium Publications, 57, 2010.

[19] M. Z. Garaev, 'Estimation of Kloosterman sums with primes and its application', Math. Notes 88:3 (2010), 365-373.

[20] D. R. Heath-Brown, 'Almost-primes in arithmetic progressions and short intervals', Math. Proc. Cambridge Philos. Soc. 83 (1978), no. $3,357-375$.

[21] M. N. Huxley, 'On the difference between consecutive primes', Invent. Math. 15 (1972), 164-170.

[22] K. Ireland and M. Rosen, A classical introduction to modern number theory, Springer, 1990.

[23] A. A. Karatsuba, 'New estimates of short Kloosterman sums', Math. Notes 88:3 (2010), 347-359. Prepared by E. A. Karatsuba, M. A. Korolev and I. S. Rezvyakova on notes and drafts of A. A. Karatsuba.

[24] A. A. Karatsuba, 'Analogues of Kloosterman sums', Izv. Math. 59:5 (1995), 971-981.

[25] A. A. Karatsuba, 'Fractional parts of functions of a special form', Izv. Math. 59:4 (1995), 721-740.

[26] M. A. Korolev, 'Incomplete Kloosterman sums and their applications', Izv. Math. 64:6 (2000), 1129-1152.

[27] M. A. Korolev, 'Short Kloosterman sums with weights', Math. Notes 88:3 (2010), 374-385.

[28] W. Luo, 'Bounds on incomplete multiple Kloosterman sums', J. Number Theory, 75 (1999), 41-46. 
[29] V. V. Prasolov, Polynomials, Algorithms and Computation in Mathematics, 11, Springer-Verlag, Berlin, 2004.

[30] I. E. Shparlinski, 'Bounds on incomplete multiple Kloosterman sums', J. Number Theory, 126 (2007), 68-73.

[31] T. Tao and V. Vu, Additive combinatorics, Cambridge Stud. Adv. Math., 105, Cambridge University Press, Cambridge, 2006.

[32] R. C. Vaughan, 'Sommes trigonométriques sur les nombres premiers', C.R. Acad. Sci. Paris Ser. A 285, 981-983. 